\title{
The EUV helium spectrum in the quiet Sun: A by-product of coronal emission?
}

\author{
V. Andretta ${ }^{1}$, G. Del Zanna ${ }^{2}$, and S. D. Jordan ${ }^{3}$ \\ ${ }^{1}$ Istituto Nazionale di Astrofisica/Osservatorio Astronomico di Capodimonte, Salita Moiariello, 16, 80131 Napoli, Italy \\ 2 Department of Applied Mathematics and Theoretical Physics, University of Cambridge, Cambridge, UK \\ ${ }^{3}$ NASA's Goddard Space Flight Center, Laboratory for Astronomy and Solar Physics, Code 682, Greenbelt, 20771 MD, USA
}

Received 12 April 2002 / Accepted 23 December 2002

\begin{abstract}
In this paper we test one of the mechanisms proposed to explain the intensities and other observed properties of the solar helium spectrum, and in particular of its Extreme-Ultraviolet (EUV) resonance lines. The so-called PhotoionisationRecombination (P-R) mechanism involves photoionisation of helium atoms and ions by EUV coronal radiation, followed by recombination cascades. We present calibrated measurements of EUV flux obtained with the two CDS spectrometers on board $\mathrm{SOHO}$, in quiescent solar regions. We were able to obtain an essentially complete estimate of the total photoionising flux in the wavelength range below $504 \AA$ (the photoionisation threshold for $\mathrm{He}$ I), as well as simultaneous measurements with the same instruments of the intensities of the strongest EUV He I and He II lines: He I $\lambda 584, \mathrm{He}$ I $\lambda 537$, and He II $\lambda 304$. We find that there are not enough EUV ionising photons to account for the observed helium line intensities. More specifically, we conclude that He II intensities cannot be explained by the P-R mechanism alone. Our results, however, leave open the possibility that the He I spectrum could be formed by the P-R mechanism, with the He II $\lambda 304$ line as a significant photoionisating source.
\end{abstract}

Key words. radiative transfer - line: formation - Sun: chromosphere - Sun: corona - Sun: UV radiation

\section{Introduction}

The question of how the helium lines are formed in the Sun has been with us since their discovery. Since helium is the second most abundant element in the Sun, and because of its apparently different fractional abundance in different regions of the solar atmosphere, especially if we include the solar wind, understanding the formation of its spectrum is of fundamental importance. In this paper we concentrate on the formation of the EUV spectrum in the quiet Sun, relying for this analysis on observations made with the Solar and Heliospheric Observatory (SOHO: Domingo et al. 1995). The particular question we address is whether the formation of the strong helium resonance lines, especially the $\mathrm{He}$ I $\lambda 584$ and $\mathrm{He}$ II $\lambda 304$ lines, can be understood in terms of reprocessed EUV coronal radiation, through photoionisation followed by recombination to the upper level of the line (either directly or in a cascade through intermediate levels). We call this process P-R, for Photoionisation-Recombination. This process is to be opposed to the standard approach to the interpretation and modelisation of EUV lines, in which collisional excitation - mainly from the ground state - is the dominant excitation mechanism.

Send offprint requests to: V. Andretta, e-mail: andretta@na.astro.it

\subsection{A brief historical overview}

A good review of the overall problem prior to the 1990's is found in Zirin (1988). Zirin reviewed a large body of evidence from both ground-based visible and radio as well as spacebased EUV observations in an effort to obtain a self-consistent picture of how all the solar helium lines are formed. In doing so, he concluded that the evidence favored the P-R mechanism for their formation. Coronal radiation was identified as the source of the required, comparatively high-energy photons short of the ionisation limits of $\mathrm{He}_{\mathrm{I}}$ and $\mathrm{He}$ II $(504 \AA$ and $228 \AA$ respectively). However, while most authors supported this view for the visible or infrared lines of neutral helium, there was considerable controversy over the formation of the principal resonance lines in particular.

A review of the alternative viewpoint, collisional excitation, in the same pre-1990s timeframe, was given by Athay (1988). In addition, C. Jordan $(1975,1980)$ noted that, if collisional excitation were the line formation mechanism of the principal resonance lines, their large excitation energy compared to their ionisation equilibrium temperature would make those lines very sensitive to non-thermal effects, such as non-maxwellian tails in the electron energy distributions. But C. Jordan also proposed that the large non-thermal velocities observed in the lineformation regions could greatly enhance the emergent intensity by exposing many of the ions to higher temperature thermal electrons, if the temperature gradient is large, as in the solar 
Transition Region (TR). We call this process "velocity redistribution", V-R, but point out that it should not be confused with use of the similar term in radiative transfer studies of optically thick lines. If velocity redistribution does indeed work as proposed, it would resolve a large discrepancy between staticatmosphere, non-LTE (NLTE) calculations (e.g. Fontenla et al. 1993) and observations from both Skylab and, more recently, SOHO (Macpherson \& Jordan 1999, henceforth: MJ99), where the observed fluxes in the principal resonance lines greatly exceed the calculated values (see below). However, it is important to note that this hypothesis is dependent upon collisional excitation dominating the line formation.

The problem prior to the 1990s was that observations were inadequate to resolve the issue for the EUV lines. Either the spatial resolution or the spectral resolution of the observations was insufficient to settle the issue, and simultaneous observations of the corresponding ionising radiation were generally lacking. With improved observations in the 1990s, the situation changed. The first mission to provide relatively high spatial and spectral resolution (5" spatial and about $55 \mathrm{m \AA}$ spectral) and at least a proxy for the photoionising radiation was the Goddard Solar Extreme Ultraviolet Research Telescope and Spectrograph (SERTS). Using data from the 1989 flight of SERTS, S. Jordan et al. (1993) concluded that collisional excitation mechanism was overwhelmingly dominant in the formation of the He II $\lambda 304$ line in the quiet Sun and probably in weak active regions, but that the issue remained open in strong active regions. Further support to these conclusions came from a subsequent study using data from later SERTS flights, by Falconer et al. (1998).

However, that conclusion was based on the assumption that the line would form near the temperature of maximum ionic abundance, $T>50000 \mathrm{~K}$, as estimated by "standard" ionisation equilibrium calculations. But, as discussed in Sect. 2, in a pure P-R scenario the helium spectrum, and in particular the He II $\lambda 304$ line, would form at much lower temperatures, where the collisional excitation rates would be dramatically reduced, and would therefore become much smaller than the photoionisation rates induced by EUV coronal radiation. In other words, Jordan et al. (1993) demonstrated that the P-R mechanism is not dominant in the TR (at $T>5 \times 10^{4} \mathrm{~K}$ ), but they could not exclude that that process could be important in the chromosphere (at $T<10^{4} \mathrm{~K}$ ). These studies, therefore, were not conclusive on the role of coronal radiation in the formation of the helium spectrum.

But by then we had entered the SOHO era. In particular, the quantity and quality of SOHO data has allowed better quantitative measurements of the properties of the solar EUV spectrum. More to the point, in MJ99 it has been shown how a standard approach based on the assumption of collisional formation of the lines, underestimates by more than an order of magnitude the intensities of the He EUV lines in the quiet Sun. That result has recently been confirmed and extended to the case of a coronal hole by C. Jordan et al. (2001).

In an attempt to resolve such a large discrepancy, assuming that collisional excitation dominated the formation of the He II $\lambda 304$ line, Andretta et al. (2000) combined observations from two SERTS flights with both SOHO and ground-based sources to assess the C. Jordan hypothesis of velocity redistribution described above. They concluded that this mechanism could at least partly explain the high observed intensity in the $304 \AA$ line in the quiet Sun, making it even more critical to determine once and for all if collisional excitation is overwhelmingly dominant in that region.

As SOHO gave us for the first time the possibility of combining observations of fluxes in the principal resonance lines and continua of neutral and ionised helium, as well as excellent images of the $304 \AA$ line to provide context, the study reported here was initiated. The methodology used in this study is discussed in detail in Sect. 2 and Appendix A, but was already applied in an embryonic form by Zirin (1975). In applying this methodology, it is important to stress that more than one formation mechanism may be at work in different regions of the atmosphere. Also, because of the large difference in optical thickness between the resonance lines and subordinate lines, the latter may easily be produced by the P-R process, even if the former are produced largely, or at least partly, by collisional excitation. An in-depth discussion of these issues, in the case of the He I spectrum, can be found in Andretta (1994) and Andretta \& Jones (1997). In the remainder of this work, we will refer to the latter paper as to AJ97.

\subsection{A note on the relevance of the problem in solar physics}

The question whether the P-R mechanism is dominant or not in the solar atmosphere, can have profound implications in the interpretation of the observations.

In particular, as discussed more in detail in Sect. 2, if the $\mathrm{P}-\mathrm{R}$ mechanism is the dominant excitation process, the formation of the helium spectrum will depend, apart from the photoionising source (the coronal EUV radiation), primarily upon the density stratification of the absorbing medium (the solar atmosphere), much less upon its temperature structure. Taking into account the relevant cross-sections and typical quiet Sun chromospheric densities, we would in fact expect helium emission to take place in a narrow and relatively homogeneous layer in the upper chromosphere (see, e.g., Zirin 1988). Again, more detailed calculations in AJ97 bear out such expectation.

Conversely, if P-R is not the dominant mechanism, the formation of the resonance helium spectrum will take place in a temperature region corresponding to the lower TR. Our understanding of the formation of the helium spectrum, then, would be intimately related to our understanding of the physics of the TR. Quite possibly, the helium spectrum could even provide important information on the structure and dynamics of that region.

With this work, we intend to contribute to such understanding, at least as far as the quiescent solar atmosphere is concerned, by using a more direct approach than the indirect clues usually produced in the past. In particular, we have taken advantage of the exceptional wavelength coverage of the SOHO EUV instruments, in particular of the Coronal Diagnostic Spectrometer (CDS, Harrison et al. 1995), while also exploiting the considerable level of accuracy and 
inter-consistency of their absolute intensity calibration. As demonstrated in the following sections, both a wide wavelength coverage and a reliable intensity calibration are essential for the test we carried out.

\section{An approach to the problem}

It is clear from the introductory overview how the problem of the formation of the EUV He spectrum is still at the stage of ascertaining which formation mechanism matches the observations at an order of magnitude level. Given this unsatisfactory state of the matter, the approach we choose to follow is then to test the simplest scenario that could explain the observations. Such a test would then indicate the direction to follow for more detailed (and complex) scenarios.

In many respects, the $\mathrm{P}-\mathrm{R}$ mechanism provides the easiest alternative to explain most of the observed properties of the solar helium spectrum. There are some indirect, often ambiguous clues both in favour and against the P-R mechanism, but much the same is true for other proposed alternatives. Therefore, applying Occam's razor, the P-R mechanism is to be preferred to the other alternatives based on less certain and/or more complicated physics, until it is demonstrated it is not compatible with the observations.

If we want to verify whether the observed EUV He intensities are compatible with the P-R mechanism, the most basic, non-trivial question to be answered, then, is whether the P-R mechanism can be considered the sole significant, or dominant source of excitation of the EUV He spectrum. More precisely, when in this paper we say that the P-R mechanism is "dominant", we mean that the contribution to the observed intensities from all the other alternative, plausible processes (mostly collisional excitation, with or without the help of some enhancement mechanism) are at least an order of magnitude smaller than the P-R contribution. Such an approach is consistent both with the findings of MJ99, and with the observational uncertainties of the method discussed in Sect. 3.

In this paper we will try to answer such a question by comparing the observed photoionising flux from coronal sources with the emerging He EUV radiation from the quiescent solar atmosphere. In the interpretation of the observations, we will reduce the number of assumptions to a minimum, in an effort to make the results of the test as model-independent as possible. In particular, we will carry out such a comparison without resorting to detailed NLTE radiative-transfer calculations, which would otherwise require realistic geometric and physical models of the solar atmosphere, a task well beyond the scope of this paper.

Following this approach, the paper is organised in two main parts:

1. A description of the test used to verify the compatibility of the P-R mechanism with observations (Sect. 2 and Appendix A). Leaving a general discussion of the method to Appendix A, in Sect. 2.2 we describe its application to the case of the EUV helium spectrum (He I and He II together), while in Sect. 2.4, we show how it is possible to restrict the same test to the case of He II emission without a significant loss of generality.

2. An application of the test (Sect. 3). After discussing in detail the rationale for using SOHO data (Sect. 3.1), we apply the test to quiescent solar regions observed in May 1997. The observations are described in Sect. 3.2, while the analysis procedures are discussed in Sect. 3.3.

The results are presented and discussed in Sect. 4, while the main conclusions are summarised in Sect. 5.

\subsection{Context physics}

The EUV wavelength range of interest for the present discussion is shown in Fig. 1, together with the relevant photoionisation cross-sections. The wavelengths of the first members of the resonance series of helium and hydrogen are marked with solid vertical lines, together with the wavelengths of the corresponding continuum limits (dashed vertical lines). For ease of comparison, Fig. 1 also shows the first-order wavelength coverage of CDS. We also note that one of the filters of the the SOHO Extreme Ultraviolet Imaging Telescope (EIT, Delaboudinière et al. 1995) is centered on the He II $\lambda 304$ line, while another SOHO EUV spectrometer, SUMER (Wilhelm et al. 1995), is capable of observing the He I $\lambda 584$ line in second order, as well as several terms of the He II Balmer series.

The helium cross-sections plotted in Fig. 1 are per $\mathrm{H}$ atom, i.e. multiplied by the abundance of helium (we have adopted the photospheric value $n(\mathrm{He}) / n(\mathrm{H})=0.085$ : Richard et al. 1998). In Fig. 1 we show the measured $\mathrm{He}^{0}$ cross-sections from West \& Marr (1976), while the Gaunt factors for $\mathrm{H}$ and $\mathrm{He}^{+}$are from Karzas \& Latter (1961).

In the inset we also show on a wider wavelength range the XUV opacity due to inner-shell photoabsorption by metals, again normalised to hydrogen and summed over all the elements $Z$ for which cross-sections were available: $\sum_{Z} \sigma_{Z} \times$ $(n(Z) / n(\mathrm{H}))$. In a neutral gas of photospheric composition, the total opacity is simply the sum of the latter component and the $\mathrm{He}^{0}$ and $\mathrm{H}$ contributions (as in, e.g., McCammon \& Morrison 1983). The cross sections data are from Henke et al. (1982) via the interpolation formulae of Bałucińska-Church \& McCammon (1992); the set of photospheric abundances adopted is from Grevesse \& Sauval (1998). In neutral media, Compton scattering, not shown here, becomes significant only above $\sim 4 \mathrm{keV}$.

The comparison shown in Fig. 1 is obviously for illustrative purposes only: for a chromospheric plasma the relative ionic fractions of the two helium ions and the ionisation state of hydrogen need to be taken into account; the same is true for the metal opacity, even though inner-shell cross sections vary little between neutrals and ions. The effect of the plasma ionisation state on the P-R mechanism will be discussed in Sects. 2.3 and 4.

\subsection{Testing the full EUV helium spectrum}

It is quite clear that coronal EUV radiation must induce a P-R effect in the solar atmosphere, at least to some extent. As mentioned in Sect. 1, in the quiet Sun coronal photons penetrate 


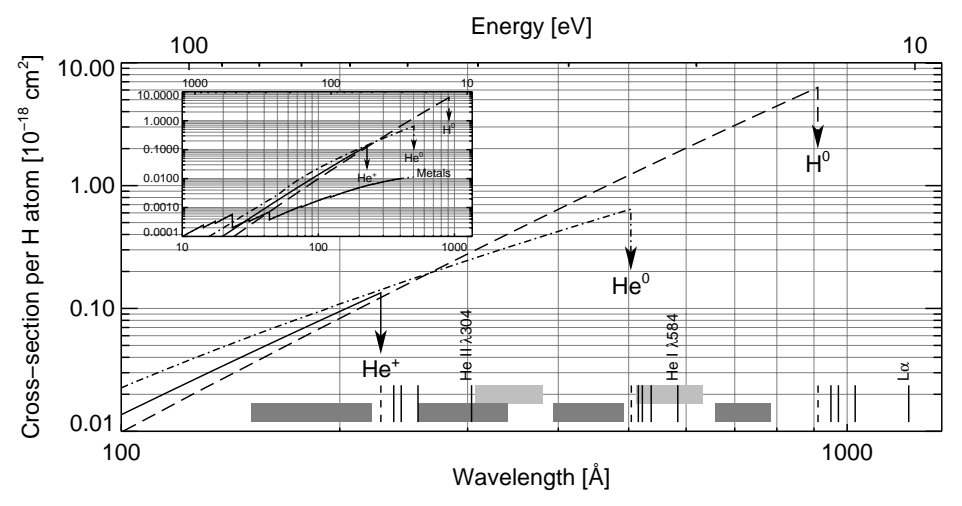

Fig. 1. Photoionisation cross sections for $\mathrm{He}^{0}$ (dot-dashes), $\mathrm{He}^{+}$(solid) and $\mathrm{H}^{0}$ (dashes), per hydrogen atom. In the inset, these cross sections are compared on a wider wavelength range to XUV photoabsorption by metals. Vertical lines at the bottom of the plot mark the wavelengths of the first resonance lines and of continuum edges for $\mathrm{He}$ and $\mathrm{H}$; the gray areas indicate the wavelength coverage (first spectral order) of the two spectrometers of SOHO/CDS: Normal Incidence, NIS (lighter gray), and Grazing Incidence, GIS (darker gray).

down to the upper chromosphere, where a narrow helium emission layer is formed. We can call this layer the "P-R layer", the analogue of a Chapman (1931) absorption layer in the Earth's ionosphere.

In this relatively cool region $\left(T<10^{4} \mathrm{~K}\right)$, collisions are very inefficient in populating the excited levels of helium atoms and ions, at energies greater respectively than $E \sim 20$ and $40 \mathrm{eV}$ from the ground level: collisional excitation rates typically depend on $T$ as $\exp (-E / k T)$. Therefore we can assume that all helium resonance photons created in a P-R layer are the result of the recombination of an electron with a helium ion or alpha particle, following photoionisation by a EUV photon. In fact, this assumption works well in the quiescent atmosphere. However, it should be noted that in denser regions (e.g. active regions), coronal photons may be absorbed in shallower, hotter layers of the atmosphere, where collisional ionisation/excitation processes may also give a significant contribution (comparable to, or even greater than P-R).

Regardless of the precise location of the P-R layer, collisional excitation processes in other, hotter layers of the atmosphere might add to that radiatively excited component (even though their simple additivity is not guaranteed, see AJ97). However, consistently with the approach based on the principle of economy we chose to follow, the hypothesis we want to test in this paper is that coronal radiation, via the P-R mechanism, is the sole significant source of excitation of helium resonance lines and continua in the quiet Sun.

In this case, we can define the efficiency of the P-R mechanism as the fraction of impinging photoionising photons reemitted back in the He resonance lines and continua. We expect this efficiency to be less than unity. We expect, that is, that the flux of coronal EUV photons (at $\lambda<504 \AA$ ) impinging on the chromosphere must be equal to, or greater than the flux of helium resonance (lines and continua) photons from the P-R layer. Thus:

$\Phi^{\mathrm{res}}[\mathrm{He} \mathrm{I}]+\Phi^{\mathrm{res}}[\mathrm{He} \mathrm{II}]<\Phi^{\mathrm{cor}}[\lambda<504]$.

In the above inequality, $\Phi^{\text {res }}[\mathrm{He}$ I $]$ and $\Phi^{\text {res }}[\mathrm{He}$ II $]$ denote, respectively, the total photon fluxes emerging from the atmosphere in the resonance series of $\mathrm{He}_{\mathrm{I}}$ (starting at $584 \AA$ ) and He II (starting at $304 \AA$ ), and from the resonance continua $(\lambda<504 \AA$ for He I, and $\lambda<228 \AA$ for He II $)$. Similarly,

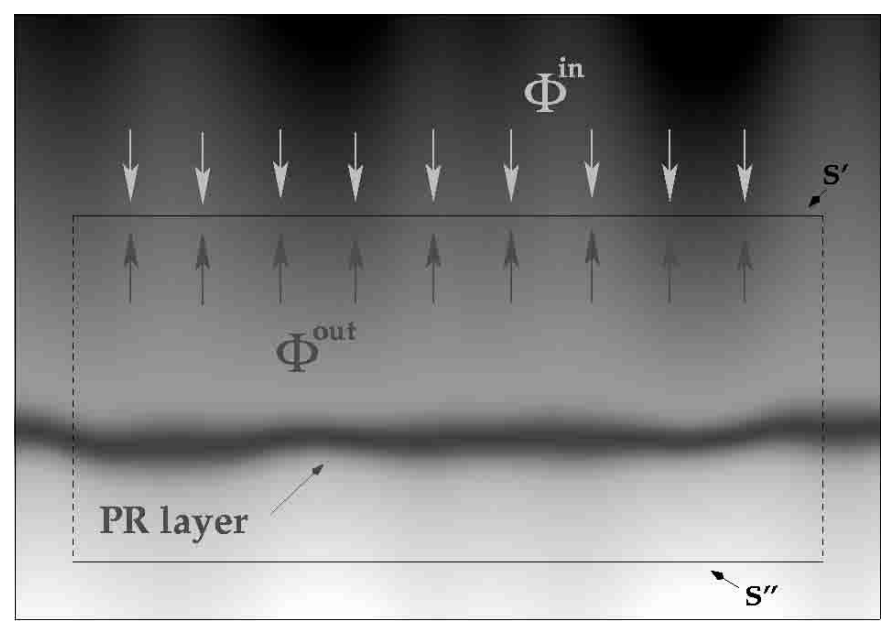

Fig. 2. Schematic geometry of the P-R mechanism in a semi-infinite, gravity-stratified atmosphere, discussed in Sect. 2 and Appendix A (density increasing towards the bottom of the sketch). The box enclosing a section of the P-R layer represents a cross section of surface $S$ across which the photon flux of Eq. (A.16) is to be evaluated. No physical or realistic scale is implied by this sketch, even though, as required in Appendix A, the vertical scale is to be considered much smaller than the horizontal size of $S$.

the notation $\Phi^{\text {cor }}[\lambda<504]$ indicates the total incident flux from all the coronal lines shortwards $504 \AA$. These fluxes, $\Phi^{\text {res }}[\mathrm{He}] \equiv \Phi^{\text {res }}[\mathrm{He} \mathrm{I}]+\Phi^{\text {res }}[\mathrm{He} \mathrm{II}]$ and $\Phi^{\mathrm{cor}}[\lambda<504]$, coincide with $\Phi^{\text {in }}$ and $\Phi^{\text {out }}$, respectively, in Fig. 2 and Appendix A, where a more formal derivation of Eq. (1) is discussed, and all the assumptions implicit in this result are spelled out.

\subsection{Some notes and caveats}

It is important to note that the derivation of Eq. (1) rests on the assumption that the absorbing medium is not embedded in the source of photoionising radiation (so that we can evaluate "incoming" and "outgoing" fluxes at surface $S^{\prime}$ : Fig. 2), and that all incident radiation is eventually absorbed (so that we can neglect flux at $S^{\prime \prime}$, as in the case of a semi-infinite atmosphere). Solar prominences, for example, may be physical instances when the application of Eq. (1) is likely not to be correct. 
Equation (1) gives equal weight to all photons capable of photoionising helium atoms and ions, whereas it would seem more intuitive to consider only those photons whose absorption cross-section is largest (photons near the photoionisation thresholds at $504 \AA$ and $228 \AA$ ). Again, this is a consequence of the assumption of complete absorption of incident radiation: a photon at $250 \AA$ is 3.5 times less likely to be absorbed by a $\mathrm{He}^{0}$ atom than one at $500 \AA$, but that only means it will travel further down (not very far, in a gravity-stratified atmosphere) before being absorbed and re-emitted, for instance in one of the resonance He I lines. By the same token, the result of Eq. (1) is independent on the abundance of helium. For the reasons expounded below, the latter quantity can affect the efficiency of the P-R mechanism, through the ratio $\mathrm{He}^{0} / \mathrm{H}$, but cannot bring it above unity.

Another point worth stressing here is that not all collisional rates need to be negligible for Eq. (1) to be valid. In fact, deexcitation rates, which have a less steep dependence on temperature than direct excitation/ionisation from ground level, may be important even at relatively low temperatures. But their effect is to remove photons from the pool of the left-hand side of Eq. (1), increasing the unbalance between $\Phi^{\text {res }}[\mathrm{He}]$ and $\Phi^{\text {cor }}[\lambda<504]$. This effect is enhanced by the presence of metastable levels, such as $\mathrm{He}^{0} 1 s 2 s^{3} \mathrm{~S}$.

In obtaining Eq. (1), some assumptions were made regarding opacity and emissivity by other elements other than helium ("background" terms; see Appendix A). Beside helium, in the wavelength range we are considering, the main absorber is hydrogen. For wavelengths below $\sim 50 \AA$, absorption by inner-shell electrons of heavier elements may also become important (Fig. 1). A rough estimate of the role of $\mathrm{H}$ can be obtained by computing the ratio of cross-sections at $504 \AA$ : $\sigma\left(\mathrm{He}^{0}\right) / \sigma(\mathrm{H})=6.1$; therefore, in a mostly-neutral plasma (and using the photospheric value $n(\mathrm{He}) / n(\mathrm{H})=0.085)$ more than a half of the photons at that wavelength would be absorbed by hydrogen. Of course, this estimate strongly depends on the relative ionisation state of $\mathrm{H}$ and $\mathrm{He}$ : a proper estimate of the effect of the opacity by hydrogen would therefore require detailed modeling (including NLTE radiative transfer). But absorption by $\mathrm{H}$ is certainly an important effect, and an effect that again goes in the direction of strenghtening the upper limit to helium emission given in Eq. (1).

Moreover, at the temperatures we are considering $(T<$ $10^{4} \mathrm{~K}$ ), photons absorbed by hydrogen are most likely reemitted beyond the $\mathrm{He}^{0}$ limit: near the edge of the Lyman continuum $(912 \AA)$ - because of the dependence as $\exp (-h v / k T)$ of its emissivity - or in the other longer-wavelength lines or continua. These photons are thus lost, as far as the helium P$\mathrm{R}$ mechanism is concerned. Furthermore, the relatively low temperature of the chromosphere ensures that emission processes other than due to $\mathrm{H}$ and $\mathrm{He}$ - emission in metallic lines, bremsstrahlung, two-photon decay from $\mathrm{H}$-like ions - give a negligible contribution in the wavelength range $\lambda<504 \AA$ (e.g. Landini \& Monsignori Fossi 1990). This justifies the assumption that the "background" emissivity is negligible in the range of interest.

\subsection{Restricting the test to ionised helium only}

In the derivation of the upper limit to helium emission of Eq. (1), all possible ways of redistributing photons among radiative transition of both $\mathrm{He}^{0}$ and $\mathrm{He}^{+}$have been taken into account. Therefore, we were able to place a constraint only on the sum of the flux from the two ions. However, a closer inspection of the rate equations reveals that, in a P-R layer, the main mechanism of interaction between the two species occurs through the transition $\mathrm{He}^{0} 1 \mathrm{~s}^{2} \longleftrightarrow 1 \mathrm{sHe}^{+}: \mathrm{He}$ II resonance photons (e.g. He II $\lambda 304$ or He II $\lambda<228$ ) can photoionise $\mathrm{He}^{0}$ atoms, and thus be converted into He I resonance photons, while, viceversa, photons in the resonance $\mathrm{He}$ I $\lambda<504$ continuum can, in principle, photoionise/excite $\mathrm{He}^{+}$ions. It is easy to realise, however, that the latter process involves only the far tail of the He I recombination continuum: given the steep dependence on wavelength of its emissivity for the temperatures we are considering, this effect is negligible. Thus, if we restrict our treatment to $\mathrm{He}^{+}$ions only, we can treat $\mathrm{He}^{0}$ atoms as another "background" absorber ("sink" of photons), exactly in the same fashion as we dealt with hydrogen.

In other words, if we make the approximation that there is no pumping of the He II resonance transitions by the He I continuum - but still allowing the reverse process, i. e. photoionisation of He I by He II radiation - we obtain:

$\Phi^{\mathrm{res}}[\mathrm{He} \mathrm{II}]<\Phi^{\mathrm{cor}}[\lambda<228]$.

The upper limit to He II emission given in the latter inequality, together with the upper limit to the total He emission of Eq. (1), is the basis for the test we are about to describe, on the compatibility of the "pure P-R" scenario with observations.

\section{The observational test}

The null hypothesis we want to test, as stated in Sect. 2, is that the $\mathrm{P}-\mathrm{R}$ mechanism is the dominant process producing the observed He emission in the quiet solar atmosphere. From an observational point of view, then, Eqs. (1) and (2) provide useful upper limits to the helium emission, with only rather weak constraints on the details of the geometry and of the transport of radiation.

No strong coronal lines overlap the main helium resonance lines: therefore, even observations with relatively modest spectral resolution would easily disentangle the photoionising radiation illuminating the chromosphere from helium emission. The only significant exception is a Six line blended with He II $\lambda 256$ (Thomas \& Neupert 1994; Brosius et al. 1998), but their contribution to terms $\Phi^{\mathrm{cor}}[\lambda<504]$ and $\Phi^{\mathrm{res}}[\mathrm{He}]$, respectively, is however small (see also Sect. 3.1). Stronger observational requirements are, on the other hand:

1. Full coverage of the range $\lambda<584 \AA$.

2. Reliable intensity calibration.

In these respects, the two CDS spectrometers are the instruments best suited for this test, as discussed below. 


\subsection{Rationale for using CDS}

\subsubsection{Wavelength coverage}

The CDS instrument is composed of a Normal Incidence (NIS) and a Grazing Incidence (GIS) Spectrometer, covering in first order the bands (see Fig. 1): 308-379 $\AA$ (NIS1); 513-633 (NIS2); 151-221 $\AA$ (GIS1); 256-341 $\mathrm{A}$ (GIS2); 393-492 (GIS3); 659-785 $\AA$ (GIS4). GIS3,4 and NIS2 also observe second order emission lines. The wavelength coverage of the GIS is sufficiently ample - covering most of the range 150-490 with its first three bands - to make it the best choice for estimating the total coronal flux impinging on the quiescent chromosphere, $\Phi^{\text {cor }}[\lambda<504]$. In Sect. 3.3 we will give an estimate of the quiet Sun coronal flux missing in GIS spectra, and show that the fluxes measured by GIS, $\Phi^{\text {cor }}[\lambda<504]$ and $\Phi^{\text {cor }}[\lambda<228]$, provide indeed a fairly complete count of the number of coronal photons below both $\mathrm{He}^{0}$ and $\mathrm{He}^{+}$photoionisation thresholds, respectively.

As far as $\mathrm{He}$ emission is concerned, $\Phi^{\text {res }}[\mathrm{He}]$, all the He II resonance series, except the He II $\lambda 304$ line, unfortunately fall in the gap between GIS1 and GIS2 or are in general unobservable. However, observations from pre-SOHO instruments (as summarised by Mango et al. 1978, for instance) give a ratio He II $\lambda 304 / \mathrm{He}$ II $\lambda 256>15$. Thus, we expect that the He II $\lambda 304$ line alone gives nearly all the total flux in the He II resonance lines.

The He II $\lambda 304$ line is so strong that the GIS does not produce reliable measurements (due to gain depression). However, this line is easily observable in second order in the NIS2 band. The higher spectral resolution in second order also allows to clearly distinguish this line from the strong Si XI $\lambda 303$ resonance line, often blended in previous instruments.

The first few lines of the resonance He I series, including the first and strongest term, the He I $\lambda 584$ line, can be accurately measured with the NIS2 (Fig. 1).

The He I and He II continua are not observed by CDS, since their thresholds are just beyond the spectral ranges of GIS1 and GIS3, respectively. It might be possible in the future to measure the tail of these continua, once the current knowledge of the edge effects on the GIS detectors has improved.

It is therefore important to estimate the contribution to $\Phi^{\mathrm{res}}[\mathrm{He}]$ from the recombination continua. We discuss this point in detail in Sect. 4.1. In short we can say that, while the precise value of the relative continuum/lines intensity ratio depends on the details of the transfer of radiation, we expect that the total photon flux in the He lines measured with CDS is lower than the total flux $\Phi^{\text {res }}[\mathrm{He}]$ by a factor of the order of two.

In summary, the two CDS detectors nicely complement each other in allowing estimates of the fluxes $\Phi^{\text {cor }}[\lambda<504]$ and $\Phi^{\text {res }}[\mathrm{He}]$ required by Eqs. (1) and (2). The main operational difficulty consists in obtaining an accurate cross-calibration between the various CDS channels, with special care on the second-order calibration of the NIS detector, essential for measuring the He II $\lambda 304$ line.

\subsubsection{Intensity calibration}

There has been a good deal of work on the part of different groups, which has been steadily converging on a consistent absolute radiometric calibration of all the instruments onboard SOHO. This has been achieved with laboratory measurements, calibration rockets, and inter-calibration studies among the different SOHO instruments. Two SOHO inter-calibration workshops have taken place, which led to a publication on the subject (Pauluhn et al. 2002). Details on the CDS calibration and its improvements over the years can be found in Del Zanna et al. (2001). These authors also provide the first complete inflight inter-calibration between all the 9 NIS and GIS channels (6 in first order and 3 in second) thereby bringing a significant update to the GIS absolute calibration, that turned out to be quite different from the one measured in laboratory. Del Zanna et al. (2001) based their relative calibration on the reference value at $584 \AA$ derived by Brekke et al. (2000) with an intercalibration between NIS and an EGS rocket flight launched in May 1997. Recently, the Brekke et al. (2000) work has been revised to take into account the effects on the NIS detector of the gain depression caused by exposures with the wide slit (Thompson 2001). The resulting new NIS calibration is similar to the previous one, but has a responsivity at $584 \AA$ that is about $15 \%$ higher. In this paper we adopt the comprehensive calibration work of Del Zanna et al. (2001) but revise the absolute values to reach consistency with the new value at $584 \AA\left(5.44 \times 10^{-4}\right.$ counts/photon $)$. This in turn has the effect of increasing the second order responsivity at $304 \AA$ to $1.56 \pm 0.4 \times 10^{-5}$.

However, it is important to realize that for our test we only need a good cross-calibration between NIS and GIS. With the improvements in the work by Del Zanna et al. (2001), the accuracy of the relative NIS/GIS calibration is estimated to be 20$30 \%$. All the recent SOHO intercalibration studies are consistent, within this uncertainty, with the CDS calibration adopted here.

As an example we consider now the CDS calibration at $304 \AA$. For the NIS in second order, the EGS and the SERTS-97 (Thomas et al. 1999) rocket flights provided two measurements of the calibration at $304 \AA$. Both rockets were calibrated on the ground against primary standards, and produced similar values of the NIS responsivity (about $1.65 \times 10^{-5}$ counts/photon). These results suffered from some uncertainties related to solar variability and the difficulty of spatial and temporal alignement with NIS. A revision of the SERTS-97 vs. NIS calibration is in progress (Thomas 2001). The second order responsivities at $304 \AA$ have recently been revised taking into account the effects of the wide-slit NIS gain depression (Thompson 2001): $1.84 \pm 0.4 \times 10^{-5}($ EGS $) ; 1.87 \pm 0.3 \times 10^{-5}$ (SERTS-97). These absolute values are only slightly higher $(15-20 \%)$ than the value adopted here.

\subsection{The observations}

The observations analysed here were taken during the period 7-13 May 1997 as part of a SOHO campaign explicitly aimed at determining the role of the P-R mechanism in the quiet 


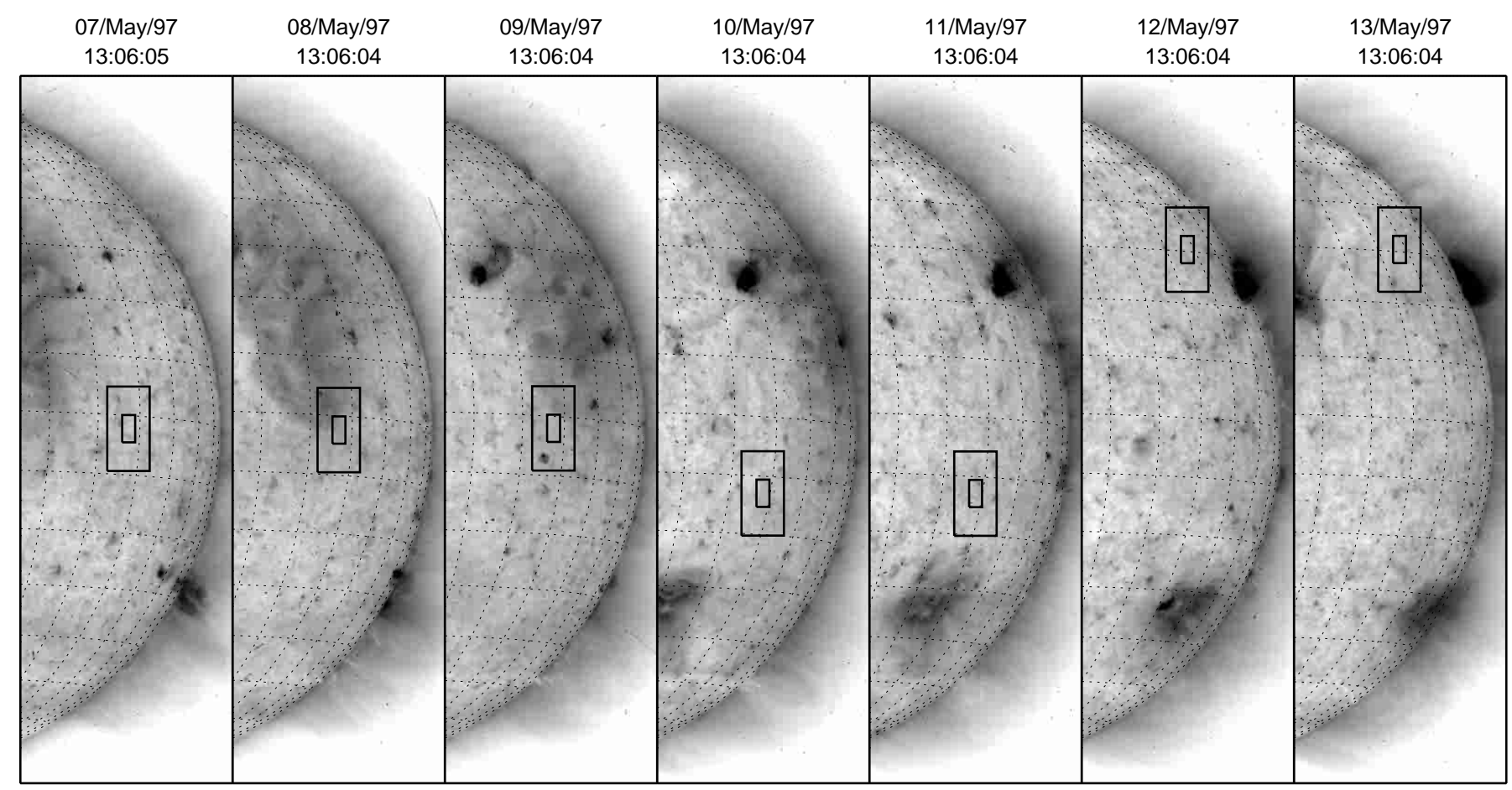

200"

Wavelength band: EIT 284

Fig. 3. Areas observed by CDS/NIS (FOV: $120 \times 240^{\prime \prime}$ ) and GIS (FOV: $40 \times 80^{\prime \prime}$ ) during the 7-13 May 1997 JOP16 campaign. The synoptic EIT 284 images shown here were taken near the end of each daily observing sequence.

Sun, and within the framework of the SOHO Joint Observing Program $\left(\mathrm{JOP}^{1}\right)$ no. 16. During this particular series of JOP16 observations, the CDS spectra were taken in coordination with another EUV SOHO spectrometer, SUMER, which provided information on the observed regions at higher temporal, spatial, and spectral resolution. A discussion of these SUMER data will be made in a follow-up study.

For planning reasons, the target region had to be selected one day prior to each observing sequence. Because of a problem in its scan mechanism, during the campaign the SUMER slit was fixed at approximately $X=+700^{\prime \prime}$ (SOHO coordinates), while still being free to move in the $\mathrm{N}-\mathrm{S}$ direction. With this constraint, we tried to select quiescent regions which during the observations would be as far as possible from active regions. Figure 3 summarises the locations observed during the campaign. The rectangular areas represents the Field-Of-View (FOV) of CDS/NIS and GIS, overlayed on one of the synoptic images taken on the same day with EIT.

The daily observing sequence for CDS consisted of alternating NIS and GIS observing programs, described below, scanning the target region. This approach minimises the effect of time variability on the total fluxes derived from the CDS spectra.

The NIS observing program used for these observations is identified by the acronym HECATRNS. This program uses the narrow slit, $2 \times 240^{\prime \prime}$, to image the target region with an E-to-W

${ }^{1}$ SOHO JOPs provide the framework for maximising the coordination between SOHO instruments, and often involve collaborations with other ground-based or space-based observatories. raster comprising 60 spectra taken at a step of $2^{\prime \prime}$ : the total area covered is then $120 \times 240^{\prime \prime}$. The exposure time of each spectrum is $46 \mathrm{~s}$; the total duration of the raster, including overheads and data transmission, is $\sim 55 \mathrm{~min}$. For telemetry reasons, data for only 14 windows of 21 pixels each in the dispersion direction were returned (4 in NIS1, and 10 in NIS2). Of these windows, three pairs were juxtaposed to cover broader multiplets or groups of lines, effectively bringing the number of windows to 11 . The windows were selected to return the profiles of the first two He I resonance lines (at $584 \AA$ and $537 \AA$ ) and the He II $\lambda 304$ line (in second order spectrum), plus some strong TR and coronal lines for context.

The acronym identifying the GIS observing program is HECATRGS. The GIS detectors are SPAN (Spiral Anode) MCP (MicroChannel Plate) detectors, described in Breeveld et al. (1992) and Breeveld (1996). The specific set of on-board Look-Up Tables (LUTs) used to produce the pixel positions along the detectors for the raw data is identified by the parameter GSET_ID. For HECATRGS, GSET_ID=42. The slit used is the no. $2: 4 \times 4^{\prime \prime}$. The program comprises 200 spectra at $10 \mathrm{mir}-$ ror (E-to-W) and 20 slit (S-to-N) positions, with a 4 " step, for an overall area covered of $40 \times 80^{\prime \prime}$. The exposure time of each spectrum is $20 \mathrm{~s}$. The total duration of the program, including overheads, is $\sim 74 \mathrm{~min}$.

In this work we choose to examine in detail the data taken on 7 and 10 May. The areas observed during these observing sequences cover quiescent areas of the Sun furthest away from active regions, at values of $\mu_{\mathrm{c}}$ (cosine of the angle from disk center) of 0.66 and 0.64 respectively, at FOV center (Fig. 3). 
The data from 11 May are similar to the data taken on 10 May, except for a rather persistent brightening crossing the middle of the GIS FOV. We will briefly discuss intensities from this feature in Sect. 4.

\subsection{Data analysis}

Standard corrections were applied to the raw NIS data. They included de-biasing, flat-fielding, corrections for gain depression effects, cosmic ray removal. A few minor corrections were applied to the GIS data, as described in Landi et al. (1999). The GIS spectra have to be deghosted (or reconstructed), before any scientific use can be made of them. Part of the counts originating from several bright lines are recorded in one or two different regions of the spectrum, thus creating one or two spurious spectral lines, called ghosts (see for details: Breeveld 1996; Landi et al. 1999; Del Zanna 1999). In some cases, ghosts appear as isolated lines, and the intensities of the lines producing them can be reconstructed. We have followed the method described in Del Zanna et al. (2001), which consists in measuring the line and ghost count rates first, and then reconstructing the GIS spectra. All the features in the GIS spectra (spectral lines identified or not, ghosts) have been considered, for a total of more than 300. A number of second order lines have been identified in the GIS3 and GIS4 spectra (for details, see Del Zanna 1999). These have been removed from the reconstructed spectra. It should be noted that for the quiet Sun observations analysed here the contribution from second order lines to the observed spectra is almost negligible, with the exception of a few lines in the GIS4 spectra (most notably the Mg IX $\lambda 368$ line), also seen in first order in the NIS spectra. The calibration has been applied as a final step, in order to convert the measured count rates in the NIS and GIS lines to intensities in photons $\mathrm{s}^{-1} \mathrm{~cm}^{-2} \mathrm{sr}^{-1}$.

As a first step, only the line intensities in the brightest lines recorded during the NIS and GIS rasters have been estimated. Monochromatic images of the FOV such as those shown in Fig. 4 have been constructed and inspected in order to judge the time variability and the presence of brightenings. In particular, for each NIS raster, the area precedently (or subsequently) observed by GIS has been considered (Fig. 4), taking into account solar rotation.

A tempting approach to the use of the series of rasters comprising the observing sequence, might involve a detailed analysis of the individual features, in order to carry out the tests described in Sect. 2 on specific quiet-Sun structures, e.g. chromospheric network boundaries and cell centers. The rather coarse time resolution of the data series, however, in conjunction with solar rotation, makes this approach very problematic. Furthermore, even if a satisfactory estimate of the observed "average" observed coronal photon intensities (along the line of sight) over, for instance, "typical" cell centers were obtained, there would still be the problem of deriving the photon flux impinging on the chromosphere from all directions - including the contribution from the surrounding network bright elements. Some additional assumptions about the threedimensional structure of the atmosphere (chromosphere and corona) would then be necessary.
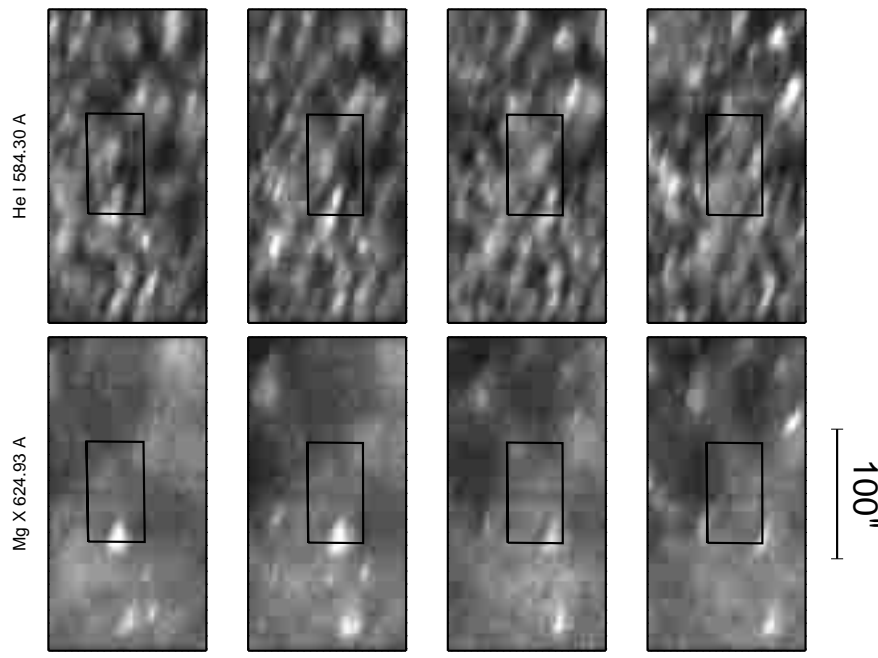

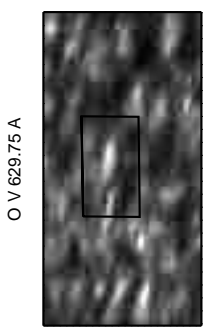

10-May-1997 07:57:05 UT

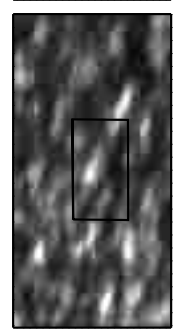

10:30:05

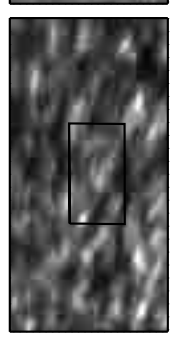

12:38:35

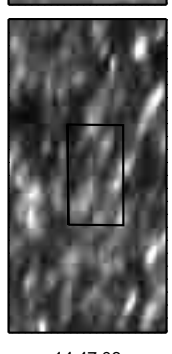

14:47:06
Fig. 4. Images obtained from selected lines in NIS spectra, for 4 rasters of the 10 May sequence. The rectangles indicate the FOV of the interleaved GIS rasters, shifted taking into account solar rotation.

In the spirit of keeping the number of assumptions to a minimum, in this paper we will only consider average spectra over the co-spatial areas observed by both NIS and GIS throughout each daily sequence, regarded as representative of average quiet Sun conditions. From the 10th of May data a small region with a brightening near the boundary of the GIS FOV (Fig. 4) has been excluded.

For each GIS and NIS raster belonging to a daily observing sequence, one average spectrum representing the co-spatial region has been computed. Count rates in the NIS and GIS lines were then obtained with a multiple line profile fitting and removal of a background (Haugan 1997). Errors on the line intensities were estimated from the errors of the line fitting parameters. Figure 5 shows the time evolution of the calibrated intensities of a few selected lines from the spatially-averaged spectra of the 10th of May. It can be seen that there is little time variability in these averaged intensities. The fact that the calibrated intensity of the Mg IX $\lambda 368$ line as observed in first (NIS) and second (GIS) order are about the same confirms the validity of the adopted cross-calibration between the two detectors. The omni-present variability of transition region lines such as $\mathrm{OV} \lambda 630$ is smoothed by the averaging over a relatively large area. The data of May 7 also show very little time variability. Once this was established, time-averaged spectra (one GIS and one NIS spectrum for each day) have been computed, and again count rates obtained with multiple line profile fitting. 


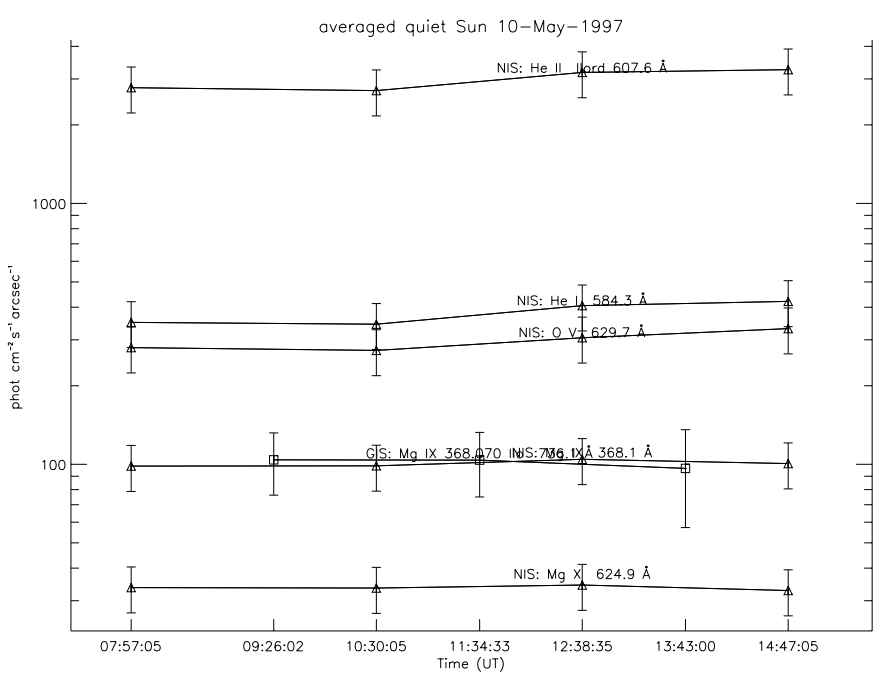

Fig. 5. Time evolution of the calibrated intensities of a few selected lines from the spatially-averaged NIS spectra of the 10th of May (triangles). The Mg IX $\lambda 368$ line has also been measured in second order in the GIS4 band (squares).

\subsubsection{Estimation of line intensities outside the observed ranges}

As already mentioned in Sect. 3.1, the observed GIS and NIS spectra cover most of the spectral range of interest here $(\lambda<$ $504 \AA$, Fig. 6). Only four wavelength ranges have incomplete or missing observed data: $1-150 \AA$; $221-257 \AA$; 340-384 $\AA$; $480-504 \AA$. The first range, $1-150 \AA$, contributes only a small fraction to the total number of photons, as explained below. The second range, 221-257 $\AA$, contains a small number of coronal lines, some of which are observed in second order in the GIS3 detector, plus a few He II lines (the strongest of which is at $256 \AA$, see discussion in Sect. 3.1). The third range, 340$384 \AA$, is mostly covered by the NIS 1 spectrum in first order (although only a few of the brightest lines have actually been extracted) and by GIS4 in second order. The fourth range, 480 $504 \AA$, only includes a few weak lines.

The "missing" line intensities have been estimated with theoretical calculations based on the CHIANTI (Dere et al. 1997) atomic database. In particular, version 3.02 (Dere et al. 2001) has been used. The CHIANTI database has been used by various authors to test the calibration of the SERTS rocket flights and the CDS instrument, and has shown a high degree of accuracy and completeness in the EUV range (see, e.g. Del Zanna 1999; Del Zanna et al. 2001, and references therein).

We have calculated the line intensities with the assumption of an optically thin collisional plasma, and performing a Differential Emission Measure (DEM) analysis following the method described in Del Zanna et al. (2001). All the observed GIS and NIS line intensities have been considered. They cover a wide range of ions and elements, and allow a good estimate of the DEM from low transition region temperatures $(\log T(\mathrm{~K})=$ 4.9; $\mathrm{C}$ III, N III) up to high temperatures $(\log T(\mathrm{~K})=6.5 ; \mathrm{Fe}$ XVI, $\mathrm{S} x \mathrm{xIV})$. Note that the majority of missing lines are emitted in the latter temperature range.

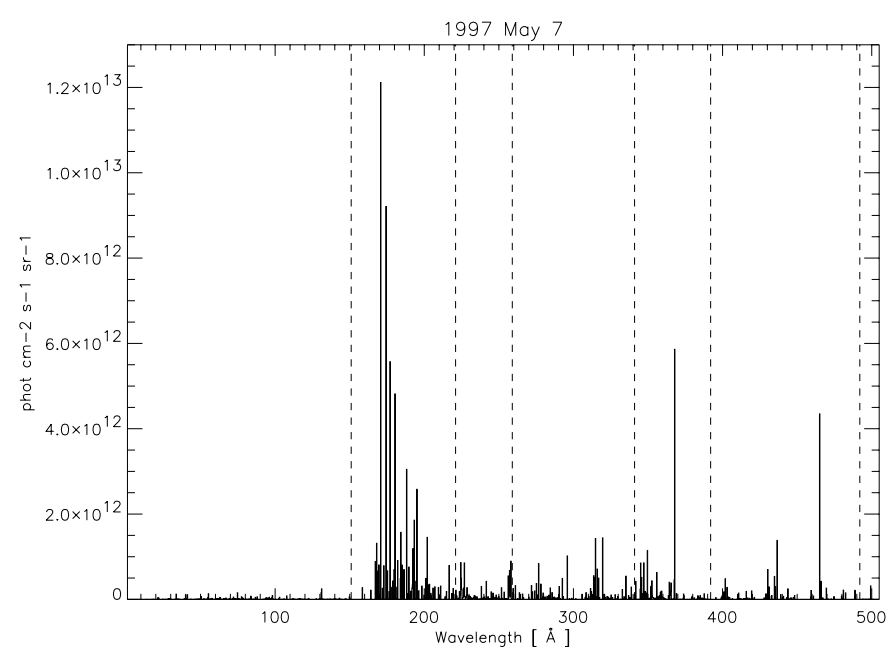

Fig. 6. EUV average spectrum relative to the quiescent region observed on May 7. Lines in the 150-221 A, 257-340 $\mathrm{\text {and }} 384-480 \AA$ ranges are from the average GIS spectrum. Intensities outside the GIS ranges are from CHIANTI theoretical calculations (see Sect. 3.3.1). Note that the strong line in the GIS2-GIS3 gap is the Mg IX $\lambda 368$ line, also observed both in second order GIS4 and first order NIS1 spectra (see also Fig. 5).

For the DEM analysis, the line emissivities have been calculated at constant density $n_{\mathrm{e}}=5 \times 10^{8} \mathrm{~cm}^{-3}$, a value derived from the excellent density-sensitive $\mathrm{Si}$ x $1347 / 356$ ratio. We note that among the "missing lines", only few are densitysensitive. These lines in the quiet Sun spectra are rather weak, and are mostly emitted at the Si x temperatures.

The photospheric abundances of Grevesse \& Sauval (1998) (with a correction to the oxygen abundance of -0.1 dex, as suggested by recent results, see Allende Prieto et al. 2001) were found to be consistent with the data in all cases. Once a DEM distribution was found, the theoretical intensities of the lines were computed and compared to the observed ones. This procedure allowed a number of useful checks to be performed.

First, we have verified that in all cases the line intensities observed by GIS were consistent (within a $20 \%$ or so) with those measured by NIS. This included transition region lines (e.g. O III, O v) observed by both instruments, and further confirmed the validity of merging the averaged GIS and NIS data.

Second, we have verified that the computed line intensities of the brightest lines were in agreement with the measured values. When lines emitted by the same ion are considered, excellent agreement (within 10\%) is found. In the other cases, differences are present, but can be explained in terms of uncertainty in the ionization fractions, as discussed in Del Zanna et al. (2001). A better agreement between theory and observations was found with the use of the old collisional ionization equilibrium computations of Arnaud \& Rothenflug (1985), compared to the most recent ones by Mazzotta et al. (1998). We therefore adopted the Arnaud \& Rothenflug (1985) calculations.

In any case, for a number of ions the emission measure approach fails in explaining the observed line intensities. This anomalous behaviour is described in detail in Del Zanna (1999) and Del Zanna et al. (2002). Fortunately, these anomalous ions fall in the GIS4 and NIS2 spectra, and not in the missing 
ranges. We can therefore be confident that the computed line intensities in the three missing ranges $(\lambda>150 \AA)$ are accurate to within $20 \%$ or so, and are complete.

We now consider the $\lambda<150 \AA$ missing range. CHIANTI version 3 (Dere et al. 2001) includes all the relevant ions (e.g. of the $\mathrm{H}$ and $\mathrm{He}$ isoelectronic sequences) and processes (e.g. inner-shell and satellite lines) that produce spectral lines in the $\mathrm{X}$-ray spectral range $(1-150 \AA)$. The coverage in this band can be considered complete for the purposes of this work, as shown in Del Zanna et al. (2001). We have performed various tests and concluded that the number of photons emitted in this band is a small fraction of the photons recorded by the GIS detectors. This is true even if Active Region (AR) observations are considered. In fact, we have taken as a test case the AR DEM derived by Schmelz et al. (1999) from YOHKOH SXT and SERTS observations. We can be confident that the high-temperature emission measure values are upper limits to the quiet Sun values, and use them at first to constrain the high$T$ tail of the DEM. However, most of the photons emitted in the 1-150 $\AA$ range are produced, even in AR spectra, by ions with peak emission between $\log T=6-6.6$. The fact that CDS is detecting lines up to $\log T=6.5$ provides a good constraint on the DEM values at those $T$ and therefore also on most of the photons emitted in the $1-150 \AA$ range.

Moreover, the higher temperature lines mainly affect the shorter wavelengths, while we have shown in Sect. 2.3 that photons below $\sim 50 \AA$ would not really matter for our test, because in chromosphere they are mostly absorbed by metals.

The various contributions entering in the estimate of the total coronal emission, including both the observed GIS spectra and the missing lines estimated through the CHIANTI package, are listed in Table 1, for the representative case of the 7 May data. The contributions of EUV continua such as freefree emission and two-photon continua from hydrogenic ions are also listed. The contribution from lines due to ions forming at temperatures below $\log T(\mathrm{~K})=5.5$ (not listed in the table) is of $4.77 \times 10^{12}$ photons $\mathrm{s}^{-1} \mathrm{~cm}^{-2} \mathrm{sr}^{-1}$, and is rather small, as expected. The most important thing to note from this table, however, is that GIS spectra do in fact allow a nearly complete count of the photons below $500 \AA$.

\subsubsection{Estimation of photon fluxes from observed intensities}

The processing of CDS data described so far gives only lineof-sight photon intensities, towards the SOHO spacecraft. With the assumption of optically thin coronal emission, these intensities also give the number of photons per unit solid angle illuminating the chromosphere, from the same direction. On the other hand, a correct application of the tests given by Eqs. (1) and (2) would require the knowledge of the radiation impinging/emitted from/to all directions, averaged over the test surface (Eq. (A.17), and Fig. 2).

Since we are considering an average quiet Sun area, we can estimate the contribution from the surrounding corona from the average limb brightening of coronal radiation. If $I(\mu)$ is the average intensity as function of the cosine of the angular distance
Table 1. Main contributions to the total coronal photoionising intensities, from the 7th of May data. The intensity of the Si XI $\lambda 303$ line is from NIS2 2nd order spectrum. Intensities are in units of $10^{12}$ photons $\mathrm{s}^{-1} \mathrm{~cm}^{-2} \mathrm{sr}^{-1}$.

\begin{tabular}{lc}
\hline \hline \multicolumn{2}{c}{ Lines - observed: } \\
$\Upsilon^{\text {cor }}[$ GIS1 $(150-221)]$ & 61.2 \\
$\Upsilon^{\text {cor }}[$ GIS2 $(257-340)]$ & 14.7 \\
$\Upsilon^{\text {cor }}[$ GIS3 $(384-480)]$ & 12.5 \\
$\Upsilon^{\text {cor }[\text { Si XI } \lambda 303]}$ & 3.86 \\
\hline \multicolumn{2}{c}{ Lines - estimated: } \\
$\Upsilon^{\text {cor }}[1-150]$ & 3.28 \\
$\Upsilon^{\text {cor }}[221-228]$ & 3.53 \\
$\Upsilon^{\text {cor }}[228-257]$ & 5.95 \\
$\Upsilon^{\text {cor }}[340-384]$ & 15.7 \\
$\Upsilon^{\text {cor }}[480-504]$ & 0.345 \\
\hline \multicolumn{1}{c}{ Others: } \\
$\Upsilon^{\text {free-free }}[1-228]$ & 2.73 \\
$\Upsilon^{\text {free-free }}[228-504]$ & 4.15 \\
$\Upsilon^{2-\text { photon}}[1-228]$ & 0.627 \\
$\Upsilon^{2-\text { photon }}[228-504]$ & 0.123 \\
\hline \multicolumn{1}{c}{ Totals: } \\
$\Upsilon^{\text {cor }}[\lambda<228]$ & 71.4 \\
$\Upsilon^{\text {cor }}[\lambda<504]$ & 129. \\
\hline \multicolumn{2}{c}{}
\end{tabular}

from disk center, $\mu$, from Eq. (A.17) (omitting mention of the frequency band, for brevity) we then have:

$\Phi^{\mathrm{in}}=\frac{2 \pi}{h v} \int_{0}^{1} \mu I(\mu) \mathrm{d} \mu$.

In terms of observations at a given $\mu_{\mathrm{c}}$ :

$\Phi^{\text {in }}=\pi f_{\mathrm{cl}}\left(\mu_{\mathrm{c}}\right) \frac{I\left(\mu_{\mathrm{c}}\right)}{h v}$,

where the correction factor, $f_{\mathrm{cl}}(\mu)$, is defined from the centerto-limb variation:

$f_{\mathrm{cl}}(\mu) \equiv 2 \int_{0}^{1} \mu^{\prime} \frac{I\left(\mu^{\prime}\right)}{I(\mu)} \mathrm{d} \mu^{\prime}$.

With this definition the correction factor is unity for a constant $I(\mu)$. An analogous correction factor can be defined for $\Phi^{\text {out }}$ as well, from the center-to-limb behaviour of the resonance He lines.

In the case of coronal limb-brightening, apart from some determination from early space missions (e.g. Withbroe 1970), not much is available in literature. More recent works mostly focus on center-to-limb variations of line widths and/or shifts (e.g. Thompson \& Brekke 2000), or on off-disk measurements. We thus have to resort to SOHO/EIT to estimate the gross properties of coronal emission as a function $\mu$. For such a quantitative estimate, we took advantage of recent improvements on the flat-fielding of EIT data (Newmark et al., in preparation).

The response of the four EIT filters is centered around $171 \AA, 195 \AA$, $284 \AA$, and $304 \AA$. For the first three filters, the main contribution to the observed intensities comes from coronal lines (respectively Fe IX $\lambda 171$, Fe XII $\lambda 195$, and Fe XV 1284). The "nominal" temperatures attributed to these images, from the peak of the temperature response, are respectively $1.3 \times 10^{6} \mathrm{~K}, 1.6 \times 10^{6} \mathrm{~K}$, and $2.0 \times 10^{6}$ 
(Delaboudinière et al. 1995), but contributions from other temperatures may be significant, especially for the EIT 195 and EIT 284 bands. The EIT 304 band is usually dominated by He II $\lambda 304$; however the Si XI $\lambda 303$ line may also be important, especially off limb.

Dividing a flat-fielded full-disk EIT image in annuli of constant $\mu$, at each value of $\mu$ we can construct an intensity histogram. The result is a two-dimensional histogram such as those shown in Fig. 7. Those histograms show a rather well defined dependence of average intensities with distance from disk center. At that stage of solar activity, with relatively few active regions on the disk, simply finding the mode of the distribution at each value of $\mu$ is usually an effective way of filtering out active regions and polar coronal holes, while the width of the central distribution (1- $\sigma$, determined from a Gaussian fit) provides error bars. In Fig. 7 we show the two-dimensional histogram of the He II (EIT 304) band, and of one of the coronal EIT bands (EIT 195). The distribution corresponding to the polar coronal holes can be seen below the main quiet-Sun distribution at $\mu<0.4$, both in the EIT 195 and (somewhat less distinct) in the EIT 304 histograms. Histograms from the other two coronal bands do not add much information, except for a somewhat greater signature of active regions at the corresponding diskcenter distances.

In the case of the coronal filter, we have superimposed a curve obtained from a very simple model of emissivity exponentially stratified with a scale height, $H$, computed for a $\mathrm{H}+\mathrm{He}$ fully ionised plasma: $H=k T / w m_{\mathrm{H}} g_{\odot}(w=0.59$ is the plasma mean molecular weight, $m_{\mathrm{H}}$ is the mass of the hydrogen atom, and $g_{\odot}$ is the solar surface gravity). Assuming an optically thin plasma, and thus from simple geometrical considerations, the resulting intensities integrated along the line of sight are:

$I(\mu, T) \propto \int_{0}^{\infty} \mathrm{d} x \exp \left\{-\frac{R_{\odot}}{H(T)}\left(\sqrt{1+2 x \mu+x^{2}}-1\right)\right\}$.

In Eq. (6) we used the "nominal" temperature of the filter, while the proportionality constant has been determined for each image by minimising the weighted $\chi^{2}$.

In the case shown in Fig. 7, the simple isothermal model given by Eq. (6) seems to give an adequate representation of the "average" properties of the on-disk quiet corona, at least for the purpose of justifying its use in Eq. (5). The same results hold for the other two coronal bands, not shown here.

The correction factors given by inserting Eq. (6) in Eq. (5) are only weakly dependent on the assumed value of $H(T)$. We adopt here as a representative temperature of the lines in the GIS range the value $T=1.0 \times 10^{6} \mathrm{~K}$, obtained from an intensity-weighted average of the temperatures of the lines. The correction factors corresponding to $\mu_{\mathrm{c}}=0.64$ and $\mu_{\mathrm{c}}=0.66$ are, respectively, $f_{\mathrm{cl}}=1.063$ and 1.09 .

In the case of the EIT 304 band, we compared the centerto-limb variation shown in Fig. 7 with the dependence found by Mango et al. (1978):

$I(\mu) \propto 1+a(1-\mu)$

with $a=0.31$ for the He II $\lambda 304$ in the quiet Sun (again, the scale factor has been found by fitting the data). For He I $\lambda 584$
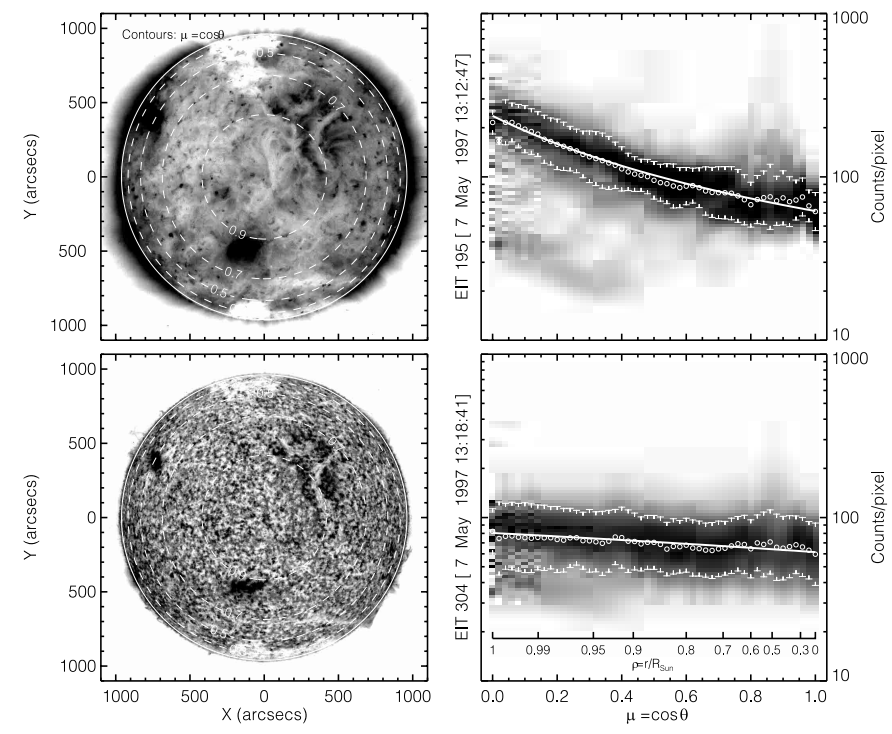

Fig. 7. Synoptic EIT images (left panels) and corresponding twodimensional histograms intensity vs. $\mu$ (right panels). In the twodimensional histograms, the mode and width of the distribution at each value of $\mu$ are indicated by circles and $\perp$ symbols, respectively. The solid lines show the fits to these values, as described in Sect. 3.3.2.

and He I $\lambda$ 537, Mango et al. (1978) give $a=0.10$ and $a=0.47$, respectively.

From Eq. (7), the correction factor is $f_{\mathrm{cl}}=(1+a / 3) /[1+$ $\left.a\left(1-\mu_{\mathrm{c}}\right)\right]$. The values of $f_{\mathrm{cl}}$ are practically unity within $1 \%$, for the actual values $\mu_{\mathrm{c}}$ of the observations analysed here (which are very near $\mu=2 / 3$ ), and for the values of $a$ given for the resonance $\mathrm{He}$ lines we are considering. In fact, at $\mu=2 / 3$, we would have $f_{\mathrm{cl}}=1$ regardless of the value of $a$. Therefore, any contamination due to the Si XI $\lambda 303$ line both in the Skylab data analysed by Mango et al. (1978) and in the broad-band EIT 304 images would not significantly affect this result, as long as a linear relationship as in Eq. (7) still fitted the data.

Incidentally, we note that if the correction factors for $\Phi^{\text {cor }}$ are nearly unity, it is also because of the particular location of the rasters we have considered (at $\mu_{\mathrm{c}} \sim 2 / 3$ ). Had we considered observations at disk center, the correction factor would have been $f_{\mathrm{cl}}=1.54$ for the coronal lines, while still being close to unity for $\Phi^{\mathrm{He}}(1.10,1.03$, and 1.16 for He II $\lambda 304$, He I $\lambda 584$, and He I 2537 , respectively) - because of the flat center-to-limb dependence of the helium resonance lines. Conversely, the coronal correction factor for the 12 and 13 May observations (at $\mu_{\mathrm{c}} \sim 0.4$ ), would have been 0.74 .

Equations (6) and (7) reproduce the "average" center-tolimb variations, such as those shown in Fig. 7, within about $10 \%$. We adopt this value as a reasonable estimate for the uncertainty in the correction factors defined in Eq. (5).

\section{Discussion of the results}

Table 2 lists the results of the analysis described in Sect. 3.3. Both the total observed intensities, $\Upsilon$, and the flux conversion factors, $f_{\mathrm{cl}}$, are listed, together with the final result: the photon fluxes required by Eqs. (1) and (2). 
Table 2. Photon intensities and fluxes from the two data sets discussed in this paper. Same units as in Table 1 . The conversion factors $\left(f_{\mathrm{cl}}\right)$ from intensities $(\Upsilon)$ to fluxes $(\Phi)$, derived as described in Sect. 3.3.2, are also listed. As discussed in the text, the tabulated helium photon fluxes are only lower limits to $\Phi^{\text {res }}[\mathrm{He} \mathrm{I}]$ and $\Phi^{\text {res }}[\mathrm{He} \mathrm{II}]$.

\begin{tabular}{|c|c|c|}
\hline & 07-May-1997 & 10-Мay-1997 \\
\hline$\mu_{\mathrm{c}}$ & 0.66 & 0.64 \\
\hline \multicolumn{3}{|l|}{ Coronal radiation: } \\
\hline$\Upsilon^{\operatorname{cor}}[\lambda<228]$ & 71.4 & 74.2 \\
\hline$\Upsilon^{\operatorname{cor}}[\lambda<504]$ & 129. & 132. \\
\hline$f_{\mathrm{cl}}^{\mathrm{cor}}$ & 1.09 & 1.063 \\
\hline$\Phi^{\mathrm{cor}}[\lambda<228]$ & 77.8 & 78.9 \\
\hline$\Phi^{\mathrm{cor}}[\lambda<504]$ & 140. & 141. \\
\hline \multicolumn{3}{|l|}{ Helium radiation: } \\
\hline$\Upsilon^{\text {res }}[\mathrm{He}$ I 2584$]$ & 15.8 & 16.1 \\
\hline$f_{\mathrm{cl}}^{\mathrm{res}}[\mathrm{He} \mathrm{I} \lambda 584]$ & 1.0 & 0.998 \\
\hline$\Upsilon^{\text {res }}[\mathrm{He}$ i 2537$]$ & 1.38 & 1.49 \\
\hline$f_{\mathrm{cl}}^{\mathrm{res}}[\mathrm{He} \mathrm{I}$ 2537] & 0.997 & 0.990 \\
\hline$\Upsilon^{\text {res }}[\mathrm{He}$ II 2304$]$ & 115. & 127. \\
\hline$f_{\mathrm{cl}}^{\mathrm{res}}[\mathrm{He}$ II $\lambda 304]$ & 0.998 & 0.993 \\
\hline$\Phi^{\mathrm{res}}[\mathrm{He} \mathrm{I}]$ & $>17.2$ & $>17.5$ \\
\hline$\Phi^{\mathrm{res}}[\mathrm{He} \mathrm{II}]$ & $>115$ & $>126$ \\
\hline$\Phi^{\mathrm{res}}[\mathrm{He}]$ & $>132$ & $>144$ \\
\hline
\end{tabular}

The relative accuracy of the GIS and NIS fluxes is subject to uncertainties in the relative calibration of the two detectors, and in the estimation of fluxes from intensities. From the analysis of Sect. 3.3, we give a conservative estimate of 30-40\% in the relative values of $\Phi^{\text {res }}[\mathrm{He}]$ and $\Phi^{\text {cor }}$.

Systematic errors in the values of the photon fluxes come, on the other hand, from an incomplete wavelength coverage of the spectral region of interest. The count of photoionising photons of coronal origin is essentially complete for $\lambda<504 \AA$ (Sect. 3.3.1), while the helium photon fluxes listed in Table 2 are only lower limits to $\Phi^{\text {res }}\left[\mathrm{He}\right.$ I] and $\Phi^{\text {res }}[\mathrm{He}$ II], because of the missing contributions from all higher resonance lines and, probably more important, from the resonance recombination continua. We briefly discuss this missing contribution to $\Phi^{\text {res }}[\mathrm{He}]$ in the following section (Sect. 4.1), before discussing the results tabulated in Table 2 . In any case, regardless of its quantitative extent, the effect concerns only the left-hand side of Eqs. (1) and (2), which therefore can still be used with the fluxes of Table 2 .

\subsection{Effect of partial coverage of the helium spectrum}

For typical chromospheric or TR temperatures, the recombination rate to the $\mathrm{He}^{+}$ground level amounts to about $1 / 3$ of the total recombinations from $\mathrm{He}^{++}$. So, as already noted by Zirin (1975), while about $2 / 3$ of the helium emission is in the form of resonance lines (mostly He II 2304 ), up to $1 / 3$ of the photons may be in the recombination continuum below $228 \AA$. Similarly, around $1 / 2$ of the recombinations to $\mathrm{He}^{0}$ are direct recombinations to the ground level.
Data for the He I recombination continuum are given by Vernazza et al. (1981): fitting the tabulated intensities for their "C" ("average") quiet-Sun component in the range 470$504 \AA$ with an exponential in frequency (i.e. assuming a constant color temperature), an integrated intensity of $9 \times$ $10^{12}$ photons $\mathrm{s}^{-1} \mathrm{~cm}^{-2} \mathrm{sr}^{-1}$ is obtained. On the other hand, Vernazza \& Reeves (1978) give for their "average" quiet Sun the intensities of the first five resonance lines (down to He I 1513), which summed together yield the value of $1.9 \times$ $10^{13}$ photons $\mathrm{s}^{-1} \mathrm{~cm}^{-2} \mathrm{sr}^{-1}$, nearly all from the He I $\lambda 584$ and He I $\lambda 537$ lines $\left(1.6 \times 10^{13}\right.$ and $1.9 \times 10^{12}$ photons s${ }^{-1} \mathrm{~cm}^{-2}$ $\mathrm{sr}^{-1}$, respectively).

For an alternative, theoretical estimate, we take as an example the calculations described in AJ97, for a set of chromospheres (the "C-nt" models of their Fig. 2) illuminated by different values of coronal EUV radiation. The contribution of the resonance continuum below $504 \AA$ to $\Phi^{\text {res }}[\mathrm{He}$ I] varies from $\sim 75-80 \%$ for a chromosphere at typical quiet Sun densities, to $\sim 40 \%$ for a chromosphere about 60 times denser.

In both cases, of course, these numbers must be taken cum grano salis, but are indicative of the fact that the resonance continua can indeed give an important contribution to the total EUV helium emission.

\subsection{The result of the test}

In both 7 May and 10 May data the total EUV coronal photoionising flux is equal, within observational uncertainties, to the sum of emerging fluxes in the He II $\lambda 304$, He I $\lambda 584$ and He I $\lambda 537$ lines. If we were able to assume that the missing contribution to $\Phi^{\mathrm{res}}[\mathrm{He}]$ is negligible, and that the efficiency of the $\mathrm{P}-\mathrm{R}$ mechanism is maximum (i.e. unity), we would conclude on the basis of Eq. (1) - that the null hypothesis being tested is compatible with the observations. Namely, we would conclude that, as stated in Sect. 2.2, a scenario in which the P-R mechanism dominates the formation of the He EUV spectrum - with other mechanisms, such as direct collisional P-R mechanism giving contributions smaller by at least an order of magnitude - could reproduce the observed helium EUV intensities.

On the other hand, the restricted test on the He II spectrum (Eq. (2)) gives a different answer. Indeed, the He II $\lambda 304$ line alone emits $50-60 \%$ more photons than all the lines and continua in the $\lambda<228$ range. Even taking into account the estimated observational uncertainty, we must conclude that He II emits at least $20 \%$ more photons than the maximum allowed by the P-R mechanism.

Without introducing further assumptions and modelling, the latter result is already significant. The result concerning the full $\mathrm{He}$ spectrum is somewhat more ambiguous, for it is clear that the He I and He II photons observed in the CDS range can quite possibly be only a fraction of, respectively, $\Phi^{\text {res }}[\mathrm{He}$ I] and $\Phi^{\text {res }}[\mathrm{He}$ II], as discussed in the previous section. Should a better characterisation of the edge effects in the GIS detectors become available, it would be interesting to add to the estimates of Table 2 the contributions of the He I and He II continua.

Assuming that in fact EUV coronal photons account only in part for the emitted helium flux in the quiet Sun, it would 
be interesting to attempt an estimate of the efficiency of the P$\mathrm{R}$ mechanism. Clearly, a quantitative estimate consistent with the data is a task well beyond the scope of the present work. Nonetheless, like in Sect. 4.1, we examined the calculations of AJ97, and found that the efficiency of the P-R mechanism on the He I spectrum, in those model chromospheres varies from $\sim 2.5 \%$ to $\sim 20 \%$, depending on the chromospheric density and on the level of coronal illumination. Should these numbers be confirmed in more detailed calculations, this would mean that the P-R mechanism, despite the apparent near equality of $\Phi^{\text {res }}[\mathrm{He}]$ and $\Phi^{\text {cor }}$, gives in reality only a minor, even negligible contribution to the formation of the EUV helium spectrum.

Finally, we note that the results of these two tests do not allow us to say much about the formation mechanism of the He I EUV spectrum. But, if we consider He II emission - however produced - as another source of photoionisation for $\mathrm{He}^{0}$, the observed He I EUV emission becomes compatible with a P-R formation: $\Phi^{\text {res }}[\mathrm{He} \mathrm{I}]<\Phi^{\mathrm{res}}[\mathrm{He} \mathrm{II}]+\Phi^{\mathrm{cor}}[\lambda<504]$, by more than an order of magnitude. The tendency of observed He I $\lambda 584$ structures to match very closely those seen in He II $\lambda 304$ images (e.g. MJ99) would even indicate that the formation of the He I spectrum is in fact dominated, via the P-R mechanism, by the latter line flux.

However, from Table 2 it seems that the He II $\lambda 304$ line, while giving an important contribution to the total flux below $504 \AA$, is by no means overwhelmingly dominant. Hence, we would expect the $\mathrm{He}$ I $\lambda 584$ line to show some correlation with coronal images as well, and that would likely degrade the excellent observed correlation with the He II $\lambda 304$ line. Moreover, there are other observational constraints - such as observed line profiles, ratio He I $\lambda 584 / \mathrm{He}$ I $\lambda 537$, color temperature of the resonance continuum (for a more in-depth discussion, see AJ97) - which are less compatible with a $\mathrm{He}_{\mathrm{I}}$ spectrum formation dominated by the P-R mechanism.

Moreover, we recall that such a straightforward comparison can be done only if we can assume that He II photoionising emission takes place above the P-R layer where the He I spectrum is produced (see Appendix A). The more realistic case of nearly co-spatial He I and He II emission would, once more, require a more detailed treatment of the transfer of radiation.

\subsection{Extending the test to other solar features}

In comparison with these quiet Sun data, the data from the 11 May brightening indicate an even more significant excess of He emission, compared with coronal radiation, both for the total $\Phi^{\text {res }}[\mathrm{He} \mathrm{I}]+\Phi^{\text {res }}[\mathrm{He}$ II $]$ radiation, and for the He II $\lambda 304$ alone. We obtain, respectively: $\Upsilon^{\text {cor }}[\lambda<228]=1.14 \times 10^{14}$, $\Upsilon^{\text {cor }}[\lambda<504]=2.15 \times 10^{14}, \Upsilon^{\text {res }}[$ He I $\lambda 584]=3.60 \times 10^{13}$, $\Upsilon^{\mathrm{res}}[\mathrm{He}$ I 2537$]=3.03 \times 10^{12}$, and $\Upsilon^{\mathrm{res}}[\mathrm{He}$ II 2304] $=2.59 \times$ $10^{14}$ photons s $\mathrm{sm}^{-1} \mathrm{sr}^{-1}$. It would be interesting (and we plan to investigate this point in the future) to verify whether this result can be extrapolated to more active areas of the solar atmosphere.

Finally, we also examined with the same criterion data for the well studied 1996 "Elephant's Trunk" coronal hole (Del Zanna \& Bromage 1999). In coronal holes, both He and coronal EUV radiation are strongly suppressed, but the latter to a greater extent (even a simple visual inspection of Fig. 7 would suffice to demonstrate this effect). In fact, considering intensities only, we obtained that $\Upsilon^{\text {res }}[\mathrm{He}]$ exceeds $\Upsilon^{\text {cor }}[\lambda<504]$ by more than a factor 2 , while $\Upsilon^{\text {res }}[\mathrm{He}$ II $]$ exceeds $\Upsilon^{\text {cor }}[\lambda<228]$ by more than a factor 4 . It is clear in this case that the P-R mechanism is not playing a significant role in the excitation of the helium spectrum in coronal holes.

\section{Conclusions}

The results presented in this paper indicate quite clearly that the excitation of the EUV helium spectrum in the quiescent solar atmosphere cannot be explained solely by a simple photoionisation-recombination process induced by coronal emission - the so-called P-R mechanism. More specifically, the observed coronal EUV flux cannot explain the formation of the He II $\lambda 304$ via the P-R mechanism. We consider this conclusion quite robust, given the minimum number of assumptions involved in the tests performed. In particular, we wish to underscore that no assumption has been made on the abundance of helium and on its variation in the solar atmosphere.

The data discussed here refer to quiescent areas, for the Sun near its minimum of activity. We have also discussed a small network brightening, drawing an analogous conclusion. Likewise, we were able to extend these results to the case of a coronal hole. It becomes now of foremost interest the possibility of performing a similar analysis in active regions: we plan to examine existing SOHO archive data in order to carry out the tests described in this paper.

On the other hand, our results do not give a definite answer regarding the formation of the He I spectrum without further assumptions. If we can assume that He II resonance emission the He II $\lambda 304$ line in particular - is produced by some other mechanism in higher layers of the atmosphere, the observed $\mathrm{He}$ I intensities are well compatible with a P-R process acting in the chromosphere, in the sense that it verifies inequalities such as Eq. (A.18). At this stage, we can only say that the problem of the formation of the He I spectrum is, if possible, even more open than the problem of the formation of the He II lines.

A possible approach to this problem requires more sophisticate modelling, including the radiative interaction between He II $\lambda 304$ and He I lines. To this respect, we note that various authors in the past (e.g. Fontenla et al. 1993; Wahlstrøm \& Carlsson 1994) have performed rather detailed calculations, concluding that the He II $\lambda 304$ line is not very important in the photoionisation of $\mathrm{He}_{\mathrm{I}}$ in the solar atmosphere. We find these arguments not very convincing: the He II $\lambda 304$ intensities computed by those authors are always much lower (even by an order of magnitude) than the observed values. Using the observed He II $\lambda 304$ intensities could significantly alter the picture.

In any case, we remark that the results presented here refer only to the resonance spectrum, i.e. only to the EUV lines. The subordinate spectrum, whose lines and continua have vastly lower optical depths, may as well be described by the P-R mechanism, or by a mixture of collisional and P-R excitation (as discussed, e.g., by AJ97, in the case of He I). 
Moreover, the hypothesis we have tested is that the P-R mechanism is the dominant mechanism in the formation of the He spectrum. We tested the hypothesis, that is, that the P-R mechanism could justify the observed EUV helium intensities, within the current observational uncertainties, without resorting to the help of other more or less plausible mechanisms. The fact that this hypothesis has been rejected, does not preclude the possibility that the P-R mechanism could give a significant contribution to the total intensities of the He EUV lines. A precise estimate of the fraction of the helium photons produced by the P-R mechanism requires an estimate of the efficiency of the process in realistic solar model atmospheres. Given the results presented in the paper, this is certainly the next step in the quest for a satisfactory resolution of the problem of the solar helium spectrum.

Finally, the fact that the P-R mechanism is definitely not the dominant formation mechanism for the He II $\lambda 304$ line and, perhaps, for the resonance HeI spectrum, calls for renewed attempts to propose a credible alternative (or additional) mechanism, considering that current methods applied to stateof-the-art solar model atmospheres fail badly to reproduce the observed intensities. Albeit formulated in a rather coarse and semi-qualitative form, the V-R mechanism first proposed by C. Jordan (1975), and more recently further developed by Andretta et al. (2000) and Smith \& Jordan (2002), seems to be, in our opinion, on the right track, together with recent improvements over the Fontenla et al. (1993) calculations, which seem to bring, with a greater extent and higher density of the TR, the calculated He II $\lambda 304$ intensities closer to the observations, even in the case of a static atmosphere (Avrett 2002) .

Investigations of the effects of non-thermal electrons were initially promising for the helium spectrum, although failing to simultaneously reproduce all the other UV emission lines (Anderson et al. 1996). However, more recent studies (Smith 2001) have given much less encouraging results even for the He lines. Nevertheless, we must note that the possibility of helium abundance variations in the solar atmosphere has rarely been considered a possible explanation. This is perhaps suprising, especially in view of the strong variations of helium abundance observed in the solar wind, or of relatively high abundance values of ambient $\mathrm{He} / \mathrm{H}$ ratio inferred in gammaray producing flares (Mandzhavidze et al. 1997). It is true that some theoretical models (Hansteen \& Leer 1997) would rather predict a depletion of helium in the TR, instead of the required strong enhancement, but the physics of such effects is sufficiently complex to merit further investigations, both on the observational and on the theoretical side.

Acknowledgements. Part of this work was carried out while one of the authors (V. A.) held a National Research Council-NASA Research Associateship at Goddard Space Flight Center. V. A. also acknowledges partial funding from NASA/GSFC Grant NCC 5-377. G. D. Z. acknowledges support from PPARC (UK) and MIUR (Italy). We are grateful to G. Severino and H. P. Jones for several useful comments and suggestions on the manuscript, as well as to Jeff Newmark for providing calibrated SOHO/EIT images used at an early stage of data analysis. We thank the staff of NASA's National Space Science Data Center for their support in obtaining the Fortran routines used to compute low-energy X-ray metal cross-sections shown in Fig. 1.
SOHO is a project of international cooperation between NASA and ESA.

\section{Appendix A: Photoionisation-recombination process in a semi-infinite, cool atmosphere}

We start off with writing the equation of radiative transfer ${ }^{2}$ averaged over all directions:

$\boldsymbol{\nabla} \cdot \boldsymbol{F}_{v}=-\chi_{v} J_{v}+\eta_{v}$

where $\chi_{v}$ and $\eta_{v}$ are the monochromatic absorption and emission coefficients, respectively, $J_{v}$ is the mean intensity: $J_{v}=$ $(4 \pi)^{-1} \int I_{\nu} \mathrm{d} \Omega$, and $\boldsymbol{F}_{v}$ is the flux: $\boldsymbol{F}_{v}=(4 \pi)^{-1} \int \boldsymbol{n} I_{\nu} \mathrm{d} \Omega(\boldsymbol{n}$ is the unit vector specifying the direction towards the solid angle $\mathrm{d} \Omega$ ). In writing Eq. (A.1) we have assumed that opacity and emissivity are isotropic.

The opacity and emissivity due the radiative transition between levels $i$ and $j$ of an atomic system $(i<j$, if we index levels with increasing energy) are:

$\chi_{i j}=n_{i} \sigma_{i j}(v)$

$\eta_{i j}=n_{j} \sigma_{j i}(v)\left(J_{v}+2 h v^{3} / c^{2}\right)$.

For bound-bound transitions, the cross-sections $\sigma$ include the absorption/emission profiles (whichever applies); $n_{i}$ is the population per unit volume of atomic level $i$. For bound-bound transitions with complete redistribution in frequency, or for bound-free continua, we have: $\sigma_{j i}(v)=$ $\sigma_{i j}(v)\left(n_{i} / n_{j}\right)^{*} \exp (-h v / k T)$, where $\left(n_{i} / n_{j}\right)^{*}$ is the ratio of the (Saha-)Boltzmann populations.

For every photon absorbed or emitted by the atom, there corresponds a radiative transition. Thus, using Eqs. (A.2) and (A.3), we can write the radiative excitation/ionisation and decay/recombination rates as:

$$
\begin{aligned}
& n_{i} R_{i j}=\int_{\Delta v_{i j}} \frac{4 \pi}{h v} \chi_{i j}(v) J_{v} \mathrm{~d} v, \\
& n_{j} R_{j i}=\int_{\Delta v_{i j}} \frac{4 \pi}{h v} \eta_{i j}(v) \mathrm{d} v,
\end{aligned}
$$

where $\Delta v_{i j}$ is the relevant frequency range for the given transition ( $v>$ threshold, for a continuum; the width of the line, for a bound-bound transition).

For a line or continuum not overlapping with other transition of the same atomic system, we can then multiply Eq. (A.1) by $4 \pi / h v$ and integrate over $\Delta v_{i j}$ to obtain:

$$
\begin{aligned}
\boldsymbol{\nabla} \cdot \boldsymbol{\Phi}\left[\Delta v_{i j}\right]= & -n_{i} R_{i j}+n_{j} R_{j i} \\
& -\int_{\Delta v_{i j}} \frac{4 \pi}{h v}\left[\chi_{b}(v) J_{v}-\eta_{b}(v)\right] \mathrm{d} v,
\end{aligned}
$$

where $\chi_{b}$ and $\eta_{b}$ are the contribution to opacity and emissivity, respectively, from sources different from the atomic system under consideration ("background" opacity/emissivity). In

\footnotetext{
${ }^{2}$ Notations and conventions mostly from Mihalas (1978), except that we do not include stimulated emission in the absorption coefficient as a negative opacity term.
} 
Eq. (A.6), the quantity $\boldsymbol{\Phi}[\Delta v]$ is the total photon flux, integrated over all the directions and over the frequency band $\Delta v$ :

$\boldsymbol{\Phi}[\Delta v] \equiv \int_{\Delta v} \frac{4 \pi}{h v} \boldsymbol{F}_{v} \mathrm{~d} v=\int_{\Delta v} \mathrm{~d} v \int \mathrm{d} \Omega n \frac{I_{v}(\boldsymbol{n})}{h v}$.

Less important in this discussion, but nevertheless useful from an observational point of view, is the total photon intensity integrated on the band $\Delta v$ along the direction $\boldsymbol{n}$; we therefore introduce a special notation for this quantity too:

$\Upsilon[\Delta v](\boldsymbol{n}) \equiv \int_{\Delta v} \mathrm{~d} v \frac{I_{\nu}(\boldsymbol{n})}{h v}$.

Finally, for the remainder of the discussion, it is also convenient to abbreviate the notation for the "background" contribution:

$\operatorname{bg}[\Delta v] \equiv \int_{\Delta v} \frac{4 \pi}{h v}\left[\chi_{b}(v) J_{v}-\eta_{b}(v)\right] \mathrm{d} v$

If transition $i \longleftrightarrow j$ overlaps with another transition of the same atom or element, $h \longleftrightarrow k$, we can still write an equation similar to Eq. (A.6):

$\boldsymbol{\nabla} \cdot \boldsymbol{\Phi}\left[\Delta v_{\cup}\right]=-n_{i} R_{i j}-n_{h} R_{h k}+n_{j} R_{j i}+n_{k} R_{k h}-\operatorname{bg}\left[\Delta v_{\cup}\right]$,

where now the integration is carried out over the set of frequencies covering both transitions: $\Delta v_{\cup} \equiv \Delta v_{i j} \cup \Delta v_{h k}$.

So far, we have merely introduced some notations, and reformulated the equation of transfer (the only assumption was the isotropy of the absorption and emission coefficients). We now introduce the equations of statistical equilibrium to express the sources and sinks of photons in terms of collisional rates.

For instance, we consider the example of an ion with $N$ bound levels (ground level indexed with 0) plus continuum. A count of all recombining photons is given by summing Eq. (A.6) for the resonance lines and the direct recombination continuum; with the adoption of the notation $\Delta v_{\cup} \equiv \bigcup_{i} \Delta v_{0 i}$, we have:

$\boldsymbol{\nabla} \cdot \boldsymbol{\Phi}\left[\Delta v_{\cup}\right]=-n_{0} \sum_{i} R_{0 i}+\sum_{i} n_{i} R_{i 0}-\operatorname{bg}\left[\Delta v_{\cup}\right]$.

With the introduction in Eq. (A.11) of the statistical equilibrium equations, we can then write:

$\boldsymbol{\nabla} \cdot \boldsymbol{\Phi}\left[\Delta v_{\cup}\right]=+n_{0} \sum_{i} C_{0 i}-\sum_{i} n_{i} C_{i 0}-\operatorname{bg}\left[\Delta v_{\cup}\right]$

where $C_{i j}$ are the collisional transition rates.

Another example is an extremely simplified 6-level model for the He ionic system: levels $\mathrm{He}^{0} 1 s^{2}{ }^{1} \mathrm{~S}, 1 s 2 s^{3} \mathrm{~S}$ and $1 s 2{ }^{1} \mathrm{P}$ (levels 0,1 and $m$ ), levels $\mathrm{He}^{+} 1 s$ and $2 p$ (levels 2 and 3 ), and $\mathrm{He}^{++}$(level 4). Transitions $2 \longleftrightarrow 3(\lambda=304 \AA)$ and $2 \longleftrightarrow 4$ $(\lambda<228 \AA)$ overlap with transition $0 \longleftrightarrow 1(\lambda<504 \AA)$. While by no means realistic, this example illustrates the case of overlapping transitions in the presence of a metastable level. The wavelength range we need to consider to account for all the recombinations, $\Delta \lambda_{\cup}$, includes the range $\lambda<504 \AA$ (which covers the two continua and He II 1304), and the wavelengths covering the profile of $\mathrm{He}$ I $\lambda 584$ :

$$
\begin{aligned}
\boldsymbol{\nabla} \cdot \boldsymbol{\Phi}[\lambda 584]=\quad & n_{1} R_{10}-n_{0} R_{01}-\operatorname{bg}[\lambda 584] \\
\boldsymbol{\nabla} \cdot \boldsymbol{\Phi}[\lambda<504]= & n_{2} R_{20}-n_{0} R_{02}+n_{3} R_{32}-n_{2} R_{23} \\
& +n_{4} R_{42}-n_{2} R_{24}-\operatorname{bg}[\lambda<504] .
\end{aligned}
$$

Summing Eqs. (A.13) and (A.14), and using the equations of statistical equilibrium, we then obtain:

$$
\begin{aligned}
\boldsymbol{\nabla} \cdot \boldsymbol{\Phi}\left[\Delta v_{\cup}\right]= & n_{0} C_{01}-n_{1} C_{10}+n_{0} C_{02}-n_{2} C_{20} \\
& +n_{2} C_{23}-n_{3} C_{32}+n_{2} C_{24}-n_{4} C_{42} \\
& +n_{0} C_{0 m}-n_{m} C_{m 0}-\operatorname{bg}\left[\Delta v_{\cup}\right] .
\end{aligned}
$$

Integrating the above equations over a volume $V$, bounded by surface $S$, we can express the photon flux across $S$ in terms of the total number of emitted or absorbed photons in the volume. Of course, these terms still depends, explicitly or implicitly, on the radiation field, so we have not really gained anything in the solution of the full NLTE radiative problem. However, if we assume that when integrated in the volume $V$ :

1. the collisional excitation and ionisation terms are negligible $\left(C_{0 i} \approx 0\right.$ in the first example, Eq. (A.12), or $C_{01}, C_{02}, C_{0 m}$, $C_{23}$ and $C_{24} \approx 0$ in Eq. (A.15));

2. the background emission is negligible $\left(\eta_{b}(v) \approx 0\right.$ : hence $\left.\operatorname{bg}\left[\Delta v_{\cup}\right] \geq 0\right)$;

so that only sinks of photons remain in the right-hand sides of the above relations, we then have:

$\iint \boldsymbol{\Phi}\left[\Delta v_{\cup}\right] \cdot \mathrm{d} \boldsymbol{S} \leq 0$.

The key point is now the choice of the boundary conditions, which depend of course on the physics and geometry of the problem. In a semi-infinite atmosphere (as in the case of the solar P-R chromospheric layer), it is convenient to separate the flux of Eq. (A.16) in fluxes through the boundary surfaces above and below the layer, as in Fig. 2 (surfaces $S^{\prime}$ and $S^{\prime \prime}$, respectively). Thus, surface $S^{\prime \prime}$ can be placed in a region where all incident photons have all been either destroyed by collisional processes or re-emitted back (perhaps after multiple absorption/re-emissions or scattering processes) through surface $S^{\prime}$. We also assume that the emission of the "cool" atmosphere below the P-R layer is negligible at the frequencies we are considering - as it would be the case of the far EUV emission from the photosphere of a late-type star - and we choose to consider a volume whose dimensions in the horizontal directions are much larger than in the vertical direction, so that we can neglect side contributions. Therefore, the largest contribution to the surface integral in Eq. (A.16) comes from the flux through surface $S^{\prime}$.

We then divide the latter term in "incoming" and "outgoing" average photon flux:

$\Phi^{\text {in,out }}[\Delta v] \equiv \frac{1}{S^{\prime}} \int_{\Delta v} \iint_{S^{\prime}} \int_{\mu<,>0} \mathrm{~d} v \mathrm{~d} \Omega \mathrm{d} S|\mu| \frac{I_{\nu}(\boldsymbol{n})}{h v}$ 
where $\mu$ is the cosine of the angle between $\boldsymbol{n}$ and $\mathrm{d} \boldsymbol{S}$, obtaining finally:

$\Phi^{\text {out }}\left[\Delta v_{\cup}\right] \leq \Phi^{\mathrm{in}}\left[\Delta v_{\cup}\right]$.

The equality in relations (A.16) and (A.18) holds only if all collisional and background terms (not just ionisation/excitation rates and emissivities) are zero. In this case, the atmosphere reemits back the same number of incident photons (a direct implication, in fact, of the adopted boundary conditions at $S^{\prime \prime}$ ), though, in general, with a different spectral distribution, as determined by the details of the transport of radiation and of the atomic structure.

\section{References}

Allende Prieto, C., Lambert, D. L., \& Asplund, M. 2001, ApJ, 556, L63

Anderson, S. W., Raymond, J. C., \& van Ballegooijen, A. 1996, ApJ, 457, 939

Andretta, V. 1994, Ph.D. Thesis, Univ. of Naples "Federico II" (Italy)

Andretta, V., \& Jones, H. P. 1997, ApJ, 489, 375

Andretta, V., Jordan, S. D., Brosius, J. W., et al. 2000, ApJ, 535, 438

Arnaud, M,. \& Rothenflug, R. 1985, A\&AS, 60, 425

Athay, R. G. 1988, ApJ, 329, 482

Avrett, E. H. 2002, private communication

Bałucińska-Church, M., \& McCammon, D. 1992, ApJ, 400, 699

Breeveld, A. A. 1996, Ph.D. Thesis, Univ. of London (UK)

Breeveld, A. A., Edgar, M. L., Smith, A., Lappington, J. S., \& Thomas, P. D. 1992, Rev. Sci. Instr., 63, 1

Brekke, P., Thompson, W. T., Woods, T. N., \& Eparvier, F. G. 2000, ApJ, 536, 959

Brosius, J. W., Davila, J. M., \& Thomas, R. J. 1998, ApJS, 119, 255

Chapman, S. 1931, Proc. Phys. Soc., 43, 26

Del Zanna, G. 1999, Ph.D. Thesis, Univ. of Central Lancashire (UK)

Del Zanna, G., \& Bromage, B. J. I. 1999, J. Geophys. Res., 104, 9753

Del Zanna, G., Bromage, B. J. I., Landi, E., \& Landini, M. 2001, A\&AS, 379, 708

Del Zanna, G., Dere, K. P., Landi, E., et al. 2001, in X-ray Astronomy 2000, ed. R. Giacconi, S. Serio, \& L. Stella ASP Conf. Ser., 234 (San Francisco: ASP)

Del Zanna, G., Landini, M., \& Mason, H. E. 2002, A\&A, 385, 968

Delaboudinière, J.-P., Artzner, G. E., Brunaud, J., et al. 1995, Sol. Phys., 162, 291

Dere, K. P., Landi, E., Mason, H. E., Monsignori Fossi, B. C., \& Young, P. R. 1997, A\&AS, 125, 149

Dere, K. P., Landi, E., Young, P. R., \& Del Zanna, G. 2001, ApJS, 134, 331

Domingo, V., Fleck, B., \& Poland, A. I. 1995, Sol. Phys., 162, 1

Falconer, D. A., Jordan, S. D., Brosius, J. W., et al. 1998, Sol. Phys., 180,179

Fontenla, J. M., Avrett, E. H., \& Loeser, R. 1993, ApJ, 406, 319
Grevesse, N., \& Sauval, A. J. 1998, Space Sci. Rev., 85, 161

Hansteen, V. H., \& Leer, E. 1997, ApJ, 482, 498

Harrison, R. A., Sawyer, E. C., Carter, M. L., et al. 1995, Sol. Phys., 162,233

Haugan, S. V. H. 1997, The Component Fitting System for IDL, CDS Software Note 47,

http://solg2 . bnsc.rl.ac.uk/swnotes/cds_swnote_47.ps

Henke, B. L., Lee, P., Tanaka, T. J., Shimabukuro, R. L., \& Fujikawa, B. K. 1982, Atomic Data and Nuclear Data Tables, 27, 1

Jordan, C. 1975, MNRAS, 170, 429

Jordan, C. 1980, Phil. Trans. R. Soc. London, Ser. A, 297, 541

Jordan, C., Macpherson, K. P., \& Smith, G. R. 2001, MNRAS, 328, 1098

Jordan, S. D., Thompson, W. T., Thomas, R. J., \& Neupert, W. M. 1993, ApJ, 406, 346

Karzas, W. J., \& Latter, R. 1961, ApJS, 6, 167

Landi, E., del Zanna, G., Breeveld, E. R., et al. 1999, A\&AS, 135, 171

Landini, M., \& Monsignori Fossi, B. C. 1990, A\&AS, 82, 229

Macpherson, K. P., \& Jordan, C. 1999, MNRAS, 308, 510

Mandzhavidze, N., Ramaty, R., \& Kozlovsky, B. 1997, ApJ, 489, L99

Mango, S. A., Bohlin, J. D., Glackin, D. L., \& Linsky, J. L. 1978, ApJ, 220,683

Mazzotta, P., Mazzitelli, G., Colafrancesco, S., \& Vittorio, N. 1998, A\&AS, 133, 403

McCammon, D., \& Morrison, R. 1983, ApJ, 270, 119

Mihalas, D. 1978, Stellar Atmospheres, 2nd edn. (San Francisco: Freeman)

Newmark, J. S., Cook, J. W., Moses, J. D., et al., in preparation

Pauluhn, A., Huber, M. C. E., \& von Steiger, R., (ed.) 2002, The Radiometric Calibration of SOHO, ISSI SR-002

Richard, O., Dziembowski, W. A., Sienkiewicz, R., \& Goode, P. R. 1998, A\&A, 338, 756

Schmelz, J. T., Saba, J. L. R., Strong, K. T., Winter, H. D., \& Brosius, J. W. 1999, ApJ, 523, 432

Smith, G. 2001, Ph.D. Thesis, Univ. of Oxford (UK)

Smith, G. R., \& Jordan, C. 2002, MNRAS, 337, 666

Thomas, R. J. 2001, private communication

Thomas, R. J., Davila, J. M., Thompson, W. T., Kent, B. J., \& Hollandt, J. 1999, in American Astronomical Society Meeting, 194, 1606

Thomas, R. J., \& Neupert, W. M. 1994, ApJS, 91, 461

Thompson, W. T. 2001, private communication

Thompson, W. T., \& Brekke, P. 2000, Sol. Phys., 195, 45

Vernazza, J. E., Avrett, E. H., \& Loeser, R. 1981, ApJS, 46, 635

Vernazza, J. E., \& Reeves, E. M. 1978, ApJS, 37, 485

Wahlstrøm, C., \& Carlsson, M. 1994, ApJ, 433, 417

West, J. B., \& Marr, G. V. 1976, Proc. R. Soc. London A, 349, 397

Wilhelm, K., Curdt, W., Marsch, E., et al. 1995, Sol. Phys., 162, 189

Withbroe, G. L. 1970, Sol. Phys., 11, 42

Zirin, H. 1975, ApJ, 199, L63

Zirin, H. 1988, Astrophysics of the Sun (Cambridge: Cambridge Univ. Press) 